\title{
Trapped Ion Mobility incorporated in LC-HRMS workflows as an integral analytical platform of high sensitivity: Targeted and untargeted 4D-metabolomics in extra virgin olive oil
}

Sofia K. Drakopoulou ${ }^{1}$, Dimitrios E. Damalas ${ }^{1}$, Carsten Baessmann ${ }^{2}$, and Nikolaos S. Thomaidis 1,*

${ }^{1}$ Laboratory of Analytical Chemistry, Department of Chemistry, National and Kapodistrian University of Athens, Panepistimiopolis Zografou, 15771 Athens, Greece

${ }^{2}$ Bruker Daltonik GmbH, Fahrenheitstr. 4, 28359 Bremen, Germany

* Corresponding author: Nikolaos S. Thomaidis

Tel: +302107274317

Fax: +30 2107274750

E-mail: ntho@chem.uoa.gr

For Submission to: Journal of Agricultural and Food Chemistry 
Figure S-1. QC charts of (A) Oleacein (EMRTM: 319.1187_5.88_251.3) in negative acquisition mode and (B) Naringenin (273.0758_6.78_163.6) in positive acquisition mode..........................................................................................

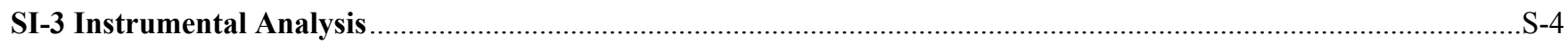

SI-4 Data Processing and Statistical Evaluation

Figure S-2. Precursor ions selection in case of LC-HRMS and LC-TIMS-HRMS. For a particular retention time range in Base Peak Chromatogram (BPC), selected at FWHM (A) different number of precursor ions are selected in LC-HRMS (B) and LC-TIMS-HRMS empowered by PASEF (C). In the case of LC-TIMS-HRMS, 5-fold more precursors approximately have been selected compared to LC-HRMS. S-5

Figure S-3. Creation of a bucket table in MetaboScape. Peak-picking parameters are selected accordingly to process data acquired from LC-TIMS-HRMS analysis (A). Intensity and peak width thresholds are implemented, while retention time and mass range are set in the same values with the analysis conditions. Adducts information is taken into account during bucket table creation, according to data acquisition mode (B). In bucket table exported, rapid isomers evaluation is also enabled within software interface, filtering the results under "isomers" keyword (C). Features of same mass measured, eluted at similar retention times but differ in terms of mobility are highlighted as potential isomers. S-6

Figure S-4. Merging example of a feature detected in both negative and positive modes, into a unified feature (A). The unified feature comprises the isotopic patterns of all different adducts detected ibn each ionization, their CCS values for each processed sample (B), as well as their respective MS/MS spectra (C) ……......................................................

SI-5 CCS-enriched target database S-8

Figure S-5. Representative examples of Apigenin and Naringenin for the acquisition of chromatographic, mobility and spectral information, used in target database development. Extracted Ion Chromatography (EIC) (A) and Extracted Ion Mobilogram (EIM) (B) of each compound were exported, as well as their MS/MS spectra (C)...................................S-8

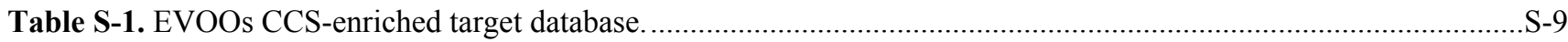

Table S-2. \%CCS error as calculated comparing negative and positive acquisition mode. ...........................................S-10

Table S-3. $\triangle$ CCS as calculated comparing CCS exported in other IMS studies. .............................................................. -11

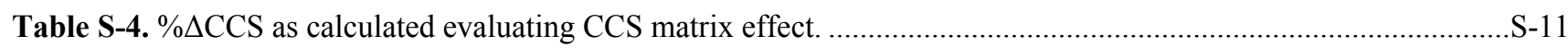

SI-6 Separation and identification of secoiridoids isomers S-13

Figure S-6. Extracted Ion Mobility (EIM) for $361.1293 \mathrm{~m} / \mathrm{z}$. Setting retention time filtering limitations ligstroside aglycone presents 7 chromatographic peaks (A), while Oleokoronal 4 chromatographic peaks respectively (B),. Different mobility profile is recorded for each respective chromatographic peak, in terms of intensity as well.

Figure S-7. Fragmentation pathways to explain $\mathrm{m} / \mathrm{z}$ detected in Ligstroside aglycone open form MS/MS spectra, in negative acquisition mode. ${ }^{7}$ Different fragments are detected depending on the part of the compound that deprotonation occurs. When deprotonation takes place at the side of the benzene ring, the main fragment detected is the 291.087 ion. On the contrary, when the compound is deprotonated at the other side of the molecule, 241.071 is produced as the main fragment.........S-14

SI-7 Holistic untargeted data treatment workflow

Figure S-8. Spectral library environment in MetaboScape software. MS/MS spectrum of the compound under identification (notified as "Query spectrum") is compared to MS/MS spectra of compounds included in the spectral library. 3 compounds of high structure similarity, Apigenin (A), Galangin (B) and Genistein (C), are presented as identification candidates, with Apigenin spectral profile best matching in the MS/MS profile of the investigated feature.

S-16

Figure S-9. Typical chromatographic profile (BPC) as acquired in negative ionization mode (A). Bioactive compounds are mainly detected in the first 12 minutes approximately of the chromatography, with the class of fatty acids following elution gradient. Representative report in Bruker MetaboScape software (B). Each feature extracted is annotated using software respective tools (e.g. analyte list) and further evaluated through annotation quality (AQ) score. The annotation quality (AQ) 
column illustrates the matching confidence in terms of accurate mass, retention time, isotopic pattern (mSigma), MS/MS fragmentation, and CCS (from left to right). The matching parameters are user-defined, with a narrow (green colour) and wide (grey colour) range of confidence annotation to be customized in each measured parameter.

S-17

SI-8 TIMS implementation in EVOOs authenticity studies.

Figure S-10. PLS-DA loading plot bounded by the first two components of the analysis in EVOOs variety discrimination study. All features included in the merged data set have been introduced in the model, resulting to distinct discrimination among all the 3 groups of variety.

S-18

Figure S-11. Most important markers in EVOOs variety discrimination as highlighted by PLS-DA VIP score. In y-axis, features detected are coded according to the format "accurate mass $(\mathrm{m} / \mathrm{z})$ retention time_CCS value", that corresponds to experimental measurements of each feature in particular. Markers of each variety are depicted, labelled at different colours, while their acquisition mode (negative/positive) is also clarified. S-18

Table S-5. Identified metabolites in EVOOs variety discrimination study based on positive and negative mode LC-TIMSTOFMS measurements, classified according to their VIP score. Features detected are coded according to the format "accurate mass (m/z)_retention time_CCS value", that corresponds to experimental measurements of each feature in particular.

Figure S-12. Number of features detected in LC-HRMS empowered by PASEF and in LC-HRMS. TIMS-PASEF incorporation in the methodology results in an increase in features number, while MS/MS spectra acquisition is also amplified.

Figure S-13. Secoiridoid isomers - markers of geographical origin, detected in negative ionization mode in correspondence with their VIP score. In y-axis, features detected are coded according to the format "accurate mass (m/z)_retention time_CCS value", that corresponds to experimental measurements of each feature in particular. Circled in red is the feature with the major contribution in the achieved discrimination.

S-20

Table S-6. Identified secoiridoid isomeric markers in EVOOs geographical origin discrimination study. Features detected are coded according to the format "accurate mass (m/z)_retention time_CCS value", that corresponds to experimental measurements of each feature in particular.

Figure S-14. Characteristic example of Ligstroside aglycone (accurate $\mathrm{m} / \mathrm{z}$ 361.1293) indicating the high sensitivity achieved through PASEF acquisition mode. Selecting a particular retention time range in Base Peak Chromatogram (BPC) in which the compound elutes (A), a large number of precursor ions are detected, Ligstroside aglycone included (B). The precursor ion of Ligstroside aglycone is selected by the quadrupole mass filter, even at 3 order of magnitude lower that the most abundant ion of the BPC (C). Reliable MS/MS spectrum of the compound is acquired, with compound's main

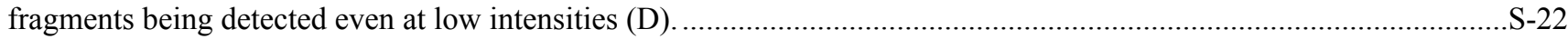

SI-9 Integrated workflow for the identification of unknowns

Figure S-15. Primary information for the two features, differentiated by their mobilities, as retrieved from MetaboScape bucket table. Chromatographic and mobility information of the feature is provided, while quick visualization of feature contribution in the discrimination is achieved through box plot chart.

S-23

Figure S-16. SmartFormula implementation for molecular formula assignment. SF3D takes into account all information of MS and MS/MS data, thus reducing significantly the number of possible candidates that comply with the matching criteria. Focusing on the class of bioactive compounds, the search is limited to compounds consisted exclusively of carbon, hydrogen and oxygen.

Figure S-17. Structure annotation was achieved via online or personal databases search, using Compound Crawler tool. 2 potential isomers are prevailed in the online database search, highlighted as spatial isomers. S-24

Figure S-18. In silico fragmentation of the compound performed using MetFrag application tool. Each fragment is pinpointed in the MS2 spectrum with a vertical red line, while the fragment is also highlighted in yellow upon compound's structure. The proposed compound best explains all fragments, recording the best matching score 1.0 (highlighted in red).

S-25 


\section{SI-1 Reagents and Standards}

All standards and reagents used were of high-purity grade ( $>95 \%)$ : catechin, ferulic acid, epicatechin, quercetin, pinoresinol, syringic acid, salicylic acid, myricetin, and eriodictyol were acquired from Sigma-Aldrich (Stenheim, Germany). Luteolin and hydroxytyrosol were purchased from Santa Cruz-Biotechnology (Santa Cruz, CA, USA), while caffeic acid, vanillin, apigenin and naringenin were purchased from Alfa Aesar (Karlsruche, Germany). Ligstroside aglycone, oleacein, oleocanthal, oleocanthalic acid, oleomissional and oleuropein aglycone were obtained from Prof. P. Magiatis laboratory (Laboratory of Pharmacognosy and Natural Products Chemistry, Faculty of Pharmacy, University of Athens, Greece). The standards had been previously isolated from olive oil extracts and their structure and purity grade were evaluated by NMR analysis. ${ }^{1}$

Methanol (MeOH) (LC-MS grade) was purchased from Merck (Darmstadt, Germany), whereas sodium hydroxide monohydrate for trace analysis $\geq 99.9995 \%$, ammonium acetate, ammonium formate and formic acid 99\% were purchased from Fluka (Buchs, Switzerland). Distilled water was provided by a Milli-Q purification apparatus (Millipore Direct-Q UV, Bedford, MA, USA). Finally, regenerated cellulose syringe filters ( $\mathrm{RC}$ filters, pore size $0.2 \mu \mathrm{m}$, diameter $15 \mathrm{~mm}$ ) were acquired from Phenomenex (Torrance, CA, USA). Stock standard solutions of individual compounds $\left(1000 \mathrm{mg} \mathrm{L}^{-1}\right)$ were prepared in $\mathrm{MeOH}$ and stored at $-20^{\circ} \mathrm{C}$ in dark glass bottles. A working mix solution of $5 \mathrm{mg} \mathrm{L}^{-1}$ was prepared for analysis by gradient dilution of the stock solutions in methanol. Isomeric compounds were analysed separately, avoiding their presence in the same mixture.

\section{SI-2 EVOO Samples and Analytical Procedures}

\section{$\underline{\text { QC charts }}$}

Quality Control (QC) samples were prepared by mixing same-volume aliquots of all olive oil sample extracts. QC charts were constructed recording analyte's intensity by the intensity of internal standard (IS). The IS used was syringaldehyde in negative acquisition mode and hesperetin in positive acquisition mode, compounds of high chemical similarity but not detected in EVOO samples. Their relative intensity (analyte intensity/IS intensity) were monitored, and their ratios to IS peak intensity were calculated to evaluate system stability, recording in all cases ratios within QC limits $( \pm 2 \mathrm{~s})$.

(A)

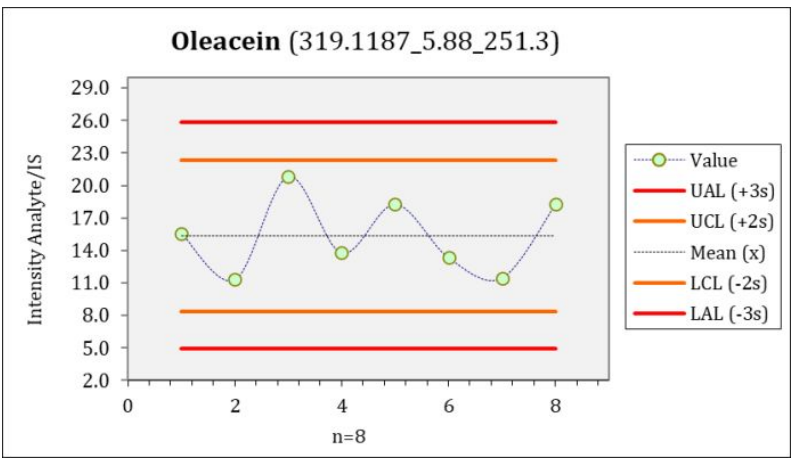

(B)

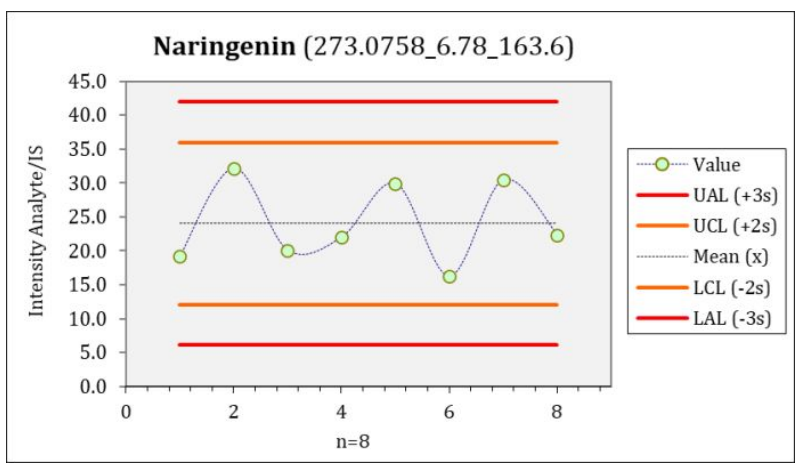

Figure S-1. QC charts of (A) Oleacein (EMRTM: 319.1187_5.88_251.3) in negative acquisition mode and (B) Naringenin (273.0758_6.78_163.6) in positive acquisition mode.

\section{SI-3 Instrumental Analysis}

Ultrahigh-performance liquid chromatography (UHPLC) of Elute LC series (Bruker Daltonics, Bremen, Germany) coupled to a hybrid trapped ion mobility-quadrupole time-of-flight (QTOF) mass spectrometer (TIMS-QTOF-MS) (timsTOF Pro, Bruker Daltonics, Bremen, Germany) was used for the analysis of the samples. For the needs of the study, in order to evaluate TIMS added value in food authenticity approach, samples were analyzed twice, once setting TIMS dimension off (typical LC-QTOF instrumentation), but also with TIMS-on making use of PASEF acquisition mode (LC-TIMS-QTOF instrumentation).

Chromatographic separation was performed on an Elute UHPLC equipped with a Solo C18 column $(2.1 \times 100 \mathrm{~mm}, 1.8 \mu \mathrm{m})$ (Bruker, Daltonics), thermostatted at $40^{\circ} \mathrm{C}$. In negative ionization mode the mobile phases consisted of water $/ \mathrm{methanol}(90: 10 \mathrm{v} / \mathrm{v}$, solvent A) and methanol (solvent B), both containing $5 \mathrm{mM}$ of ammonium acetate. In positive ionization mode the mobile phases consisted of water/methanol 90/10 (solvent A) and methanol (solvent B), both containing $5 \mathrm{mM}$ ammonium formate and $0.01 \%$ formic acid. A gradient elution program was applied in both ionization modes, starting with $1 \% \mathrm{~B}$ (flow rate of $0.2 \mathrm{~mL} \mathrm{~min}{ }^{-1}$ ) for 1 min, which was increased to $39 \%$ in 2 min and then to $99.9 \%$ (flow rate of $0.4 \mathrm{~mL} \mathrm{~min}^{-1}$ ) in another $11 \mathrm{~min}$. Then, B was kept constant for $2 \mathrm{~min}$ (flow rate of $0.48 \mathrm{~mL} \mathrm{~min}^{-1}$ ); initial chromatographic conditions were restored through re-equilibration of the column for 3 min. The injection volume was set to $5 \mu \mathrm{L}$. 
TIMS-QTOF-MS system was equipped with an electrospray ionization interface (ESI), operating in positive and negative mode. Ionization parameters in both modes included a capillary voltage of $3500 \mathrm{~V}$, a nebulizer gas pressure of 2 bar $\left(\mathrm{N}_{2}\right)$, drying gas flow at $8 \mathrm{~L} \mathrm{~min}^{-1}$, an end-plate offset of $500 \mathrm{~V}$ and a dry temperature of $200{ }^{\circ} \mathrm{C}$. A QTOF external calibration was performed daily with the manufacturer's solution. A mass scan range of $50-1000 \mathrm{~m} / \mathrm{z}$ was set. In TIMS-QTOF system operated in PASEF scan mode, $1 / \mathrm{k}_{0}$ range of $0.45-1.80 \mathrm{Vs} \mathrm{cm}^{-2}$ was selected, with medium-sized bioactive compounds detected among this mobility range. A duty cycle of $100 \%$ can be achieved thanks to dual TIMS setup, given the fact that accumulation and ramp times are kept stable. ${ }^{2}$ Thus, in our analysis accumulation and ramp time was set at $100 \mathrm{~ms}$ each, while a total cycle of $0.32 \mathrm{sec}$ was also selected resulting in one full TIMS-MS scan and two PASEF MS/MS scans. TIMS ion charge control was set to 5e6. TIMS dimension was calibrated linearly using two selected ions from ESI Low Concentration Tuning Mix (Agilent Technologies, USA) [m/z, 1/ $\mathrm{k}_{0}$ : (301.998139, 0.6678 Vs $\left.\left.\mathrm{cm}^{-2}\right),\left(601.979077,0.8782 \mathrm{Vs} \mathrm{cm}^{-2}\right)\right]$ in negative mode and $\left[\mathrm{m} / \mathrm{z}, 1 / \mathrm{k}_{0}:\left(322.048121,0.7363 \mathrm{Vs} \mathrm{cm}^{-2}\right),(622.028960,0.9915 \mathrm{Vs}\right.$ $\left.\mathrm{cm}^{-2}\right)$ ] in positive mode. The mobility for mobility calibration was taken from the CCS compendium. ${ }^{3}$

\section{SI-4 Data Processing and Statistical Evaluation}

\section{Targeted Workflow}

DataAnalysis 5.2 software (Bruker Daltonics) was used as a first step of data evaluation in the targeted approach applied. The total of 22 bioactive compounds were analyzed using LC-TIMS-QTOF, as an initial assessment of the applied methodology. Information retrieved regarding retention time, CCS values and fragments was exported and summarized in the target database. Secoiridoids isomers initial evaluation, in terms of separation and identification, was also performed within the software interface. Extracted ion chromatograms and mobilograms (EIC and EIM respectively) were imported for targeted compounds (e.g. oleuropein and ligstroside aglycone), while isomers' spectral profile was also assessed.

\section{$\underline{\text { Un-targeted Workflow }}$}

Un-targeted data treatment was performed using MetaboScape ${ }^{\circledR} 4.0$ software (Bruker Daltonics), compatible with both LC-QTOF and LC-TIMS-QTOF data. MetaboScape 4.0 is able to process data from both LC-QTOF and LC-TIMS-MS systems. Data processing from LC-QTOF analysis was performed with "T-ReX 3D", with the algorithm taking into account 3 particular parameters through peak-picking step, namely retention time, mass to charge ratio $(\mathrm{m} / \mathrm{z})$ and intensity. Respectively, in LC-TIMS-QTOF data, T-ReX 4D was applied, co-calculating CCS in the peak-picking step among the other aforementioned parameters. A number of peak-picking parameters were selected for reliable data extraction; intensity threshold was set at 2000 counts while minimum peak length was adjusted at 10 spectra for PASEF-acquisition data and 5 spectra for data obtained from LC-QTOF analysis (also DDA mode). Peak length (expressed in spectra) was optimized for LC-TIMS-HRMS (PASEF) and LC-HRMS accordingly. As depicted in Figure S-2, thanks to PASEF parallel accumulation, precursor ions selection and subsequent fragmentation are significantly increased, with more MS spectra being acquired and included per chromatographic peak. Under the same retention time range (selected at full width at half maximum (FWHM) of the Base Peak Chromatogram (BPC) in both LC-TIMS-HRMS (PASEF) and LC-HRMS), 5-fold more precursors ions approximately have been selected in the case of LC-TIMS-HRMS (PASEF) compared to LC-HRMS.

(A)

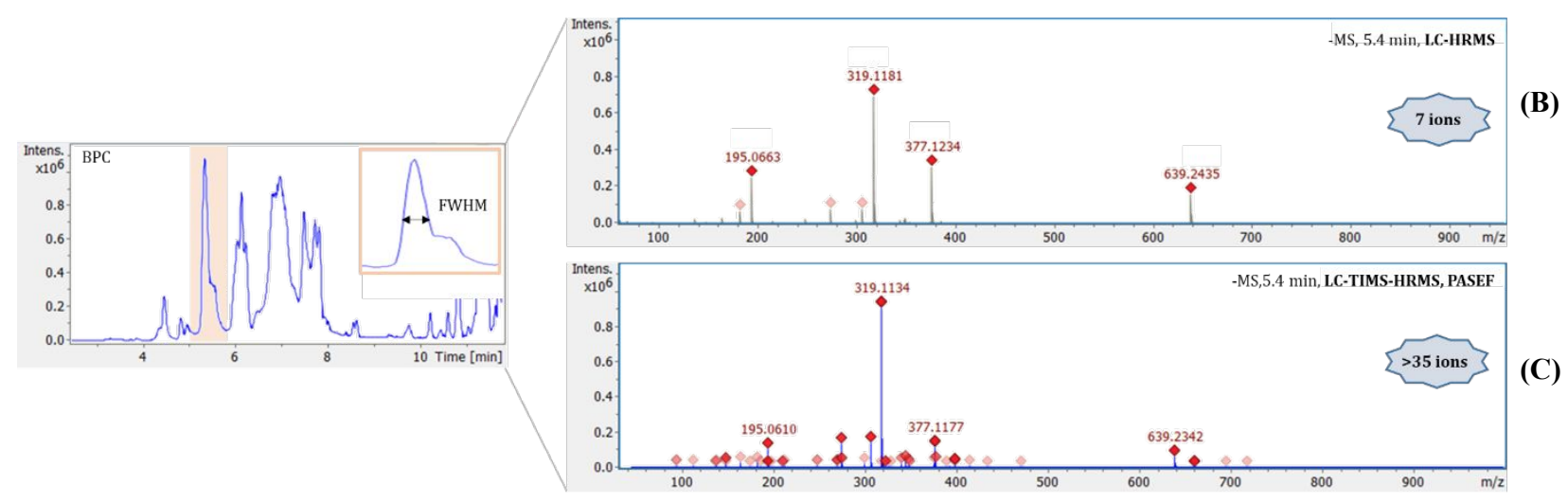

Figure S-2. Precursor ions selection in case of LC-HRMS and LC-TIMS-HRMS. For a particular retention time range in Base Peak Chromatogram (BPC), selected at FWHM (A) different number of precursor ions are selected in LC-HRMS (B) and LC-TIMS-HRMS empowered by PASEF (C). In the case of LC-TIMS-HRMS, 5-fold more precursors approximately have been selected compared to LCHRMS 
Mass recalibration was automatically performed for each sample, using sodium formate as internal calibration solution. The same calibrant was used in external calibration that preceded instrumental analysis. Retention time and mass range were set in the same values with the analysis conditions (retention time range: $0-15 \mathrm{~min}$, mass range: $50-1000 \mathrm{~m} / \mathrm{z}$ ). Primary ion detection in each acquisition mode was also selected, along with adducts ions (e.g. $\left.[\mathrm{M}+\mathrm{Na}]^{+},[\mathrm{M}+\mathrm{K}]^{+}\right)$and other occurring ions $\left(\mathrm{e} . \mathrm{g} .\left[\mathrm{M}+\mathrm{H}-\mathrm{H}_{2} \mathrm{O}\right]^{+}\right.$, $\left.[2 \mathrm{M}+\mathrm{H}]^{+}\right)$. For positive mode, $[\mathrm{M}+\mathrm{H}]^{+}$was selected as primary ion, while $[\mathrm{M}-\mathrm{H}]^{-}$was selected in negative mode respectively. All the extracted information through peak-picking step, was summarized in a generic peak list, called "bucket table", within the software interface. The bucket table included all the features extracted by the algorithm. Each feature encompassed information over the retention time, $\mathrm{m} / \mathrm{z}$, adducts detected, intensity and CCS values, in the case of LC-TIMS-QTOF data. The creation of the bucket table is depicted in Figure S-3.

(A)

\section{Data Acquisition}

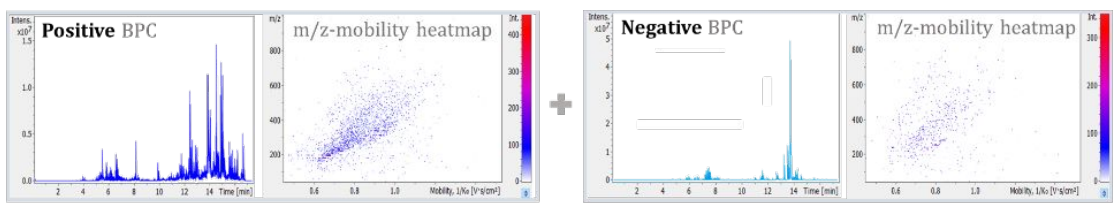

MetaboScape

T-ReX algorithm

\section{Data pre-processing}

(peak detection, calibration, filtering, bucketing)

(B)

\begin{tabular}{|c|c|c|}
\hline \multicolumn{3}{|c|}{$\begin{array}{l}\text { T-ReX Configuration } \\
\text { Set parameters for detection of molecular features. }\end{array}$} \\
\hline \multicolumn{3}{|l|}{ BucketTable } \\
\hline \multicolumn{3}{|l|}{ Name: Negative Acquisition Mode } \\
\hline \multicolumn{2}{|l|}{ Peak Detection } & Calibration \\
\hline Intensity Threshold: & counts & $\square$ Lock Mass Calibration: \\
\hline Minimum Peak Length: & \multirow[t]{2}{*}{ spectra } & RT auto-detect \\
\hline Feature Signal: Intensity & & $\square$ Mass exclusion: \\
\hline \multicolumn{3}{|l|}{ Recursive Feature Extraction } \\
\hline \multicolumn{2}{|l|}{ Minimum Peak Length (recursive): } & spectra \\
\hline
\end{tabular}
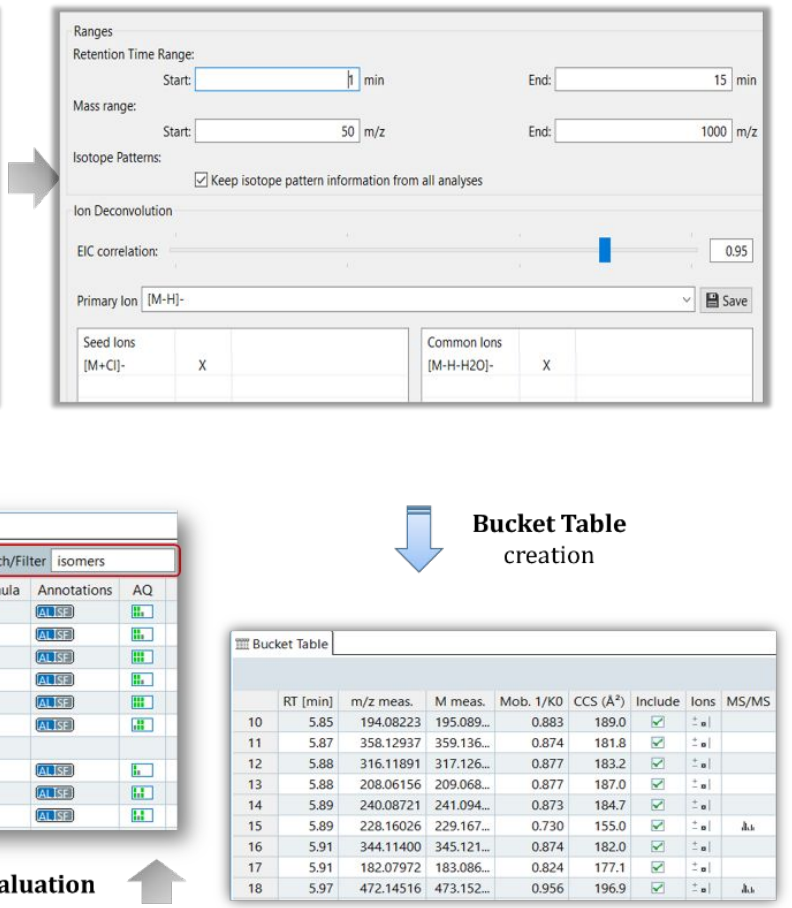

creation

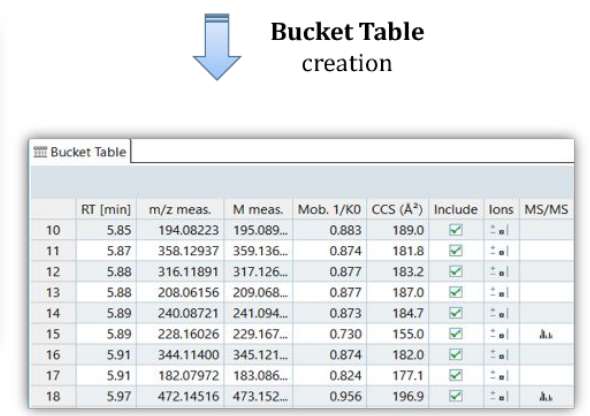

Rapid isomers evaluation

Figure S-3. Creation of a bucket table in MetaboScape. Peak-picking parameters are selected accordingly to process data acquired from LCTIMS-HRMS analysis (A). Intensity and peak width thresholds are implemented, while retention time and mass range are set in the same values with the analysis conditions. Adducts information is taken into account during bucket table creation, according to data acquisition mode (B). In bucket table exported, rapid isomers evaluation is also enabled within software interface, filtering the results under "isomers" keyword (C). Features of same mass measured, eluted at similar retention times but differ in terms of mobility are highlighted as potential isomers. 
To process data obtained from different ionization modes, two different buckets tables were created corresponding to negative and positive ionization modes. However, some features were detected in both polarities. In order to avoid features overlapping in the statistical evaluation, features detected in both ionizations were merged into a unified feature. The two bucket tables were merged, with a retention time (RT) tolerance of $2 \mathrm{sec}$ being set as a threshold. Merging of the buckets was performed only if the RT difference between the buckets was below the RT tolerance value. Information of different adducts derived from each acquisition mode were combined in one feature, as well. The unified feature included all the different adducts isotopic patterns, as well as their respective MS/MS spectra and CCS values (Figure S-4). This information was available for every processed sample.

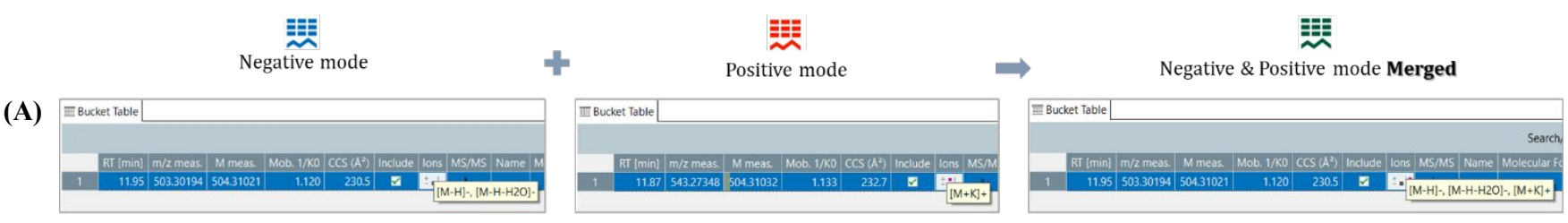

(B)

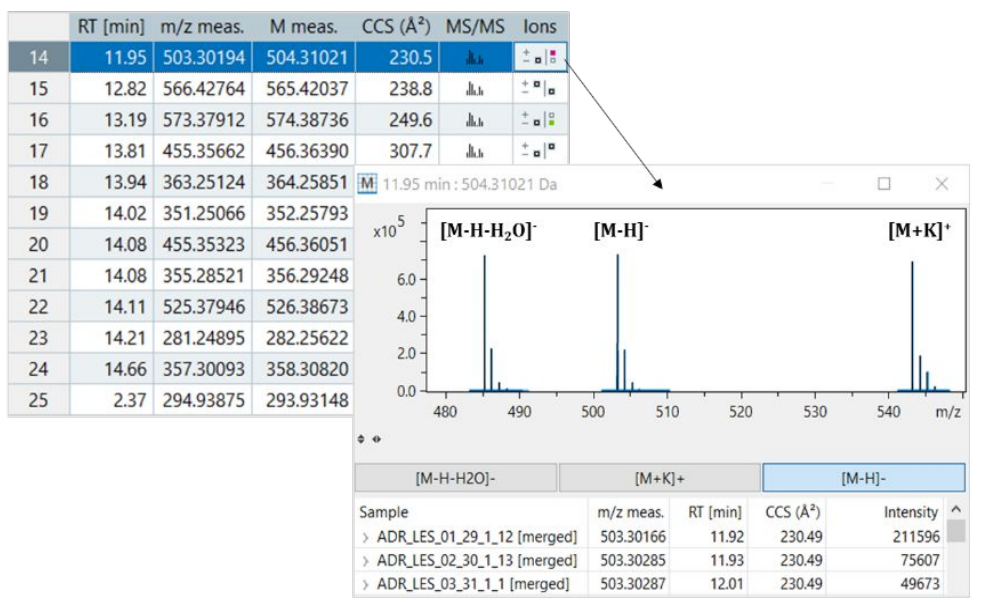

(C)

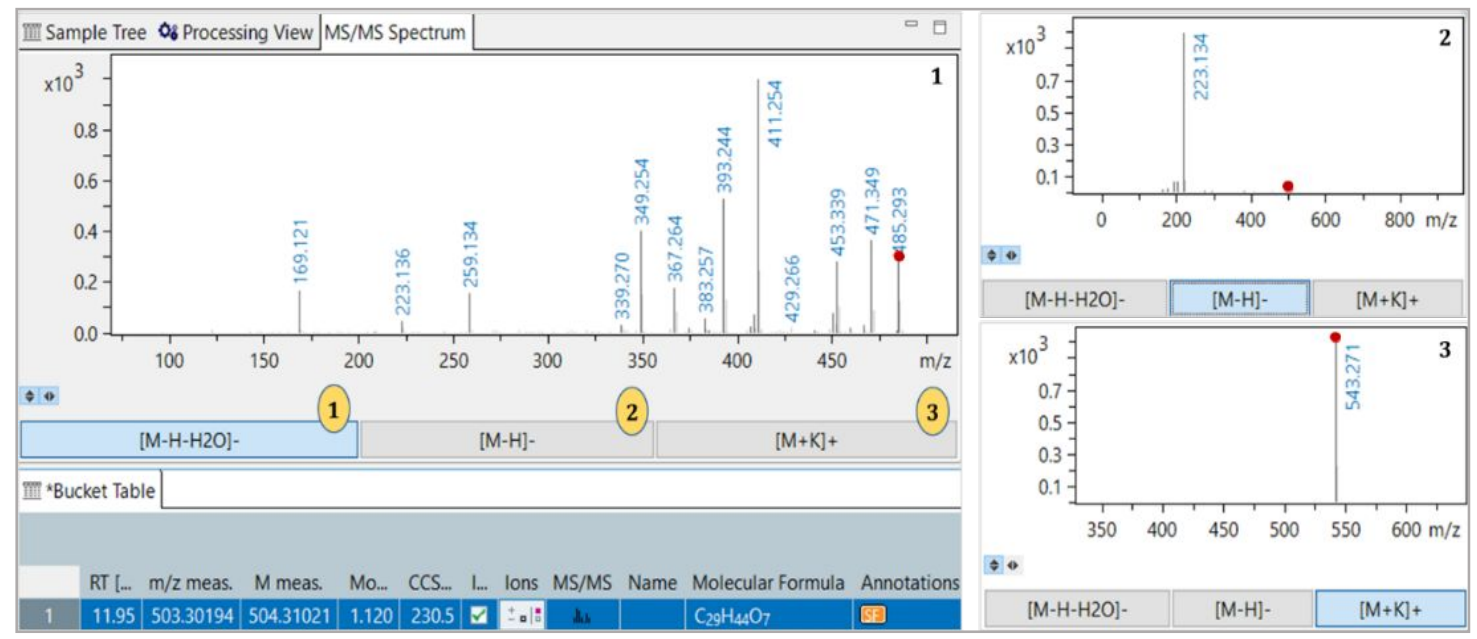

Figure S-4. Merging example of a feature detected in both negative and positive modes, into a unified feature (A). The unified feature comprises the isotopic patterns of all different adducts detected ibn each ionization, their CCS values for each processed sample (B), as well as their respective MS/MS spectra (C). 


\section{SI-5 CCS-enriched target database}

The database comprised a total of 22 bioactive compounds often occurring in olive oil. Table S-1 summarizes all information included regarding compound's name, neutral molecular formula, ions detected, retention time (min), ion mobility expressed in $\operatorname{CCS}_{\mathrm{N} 2}\left(\mathrm{~A}^{2}\right)$, fragmentation ions and their elemental formulas.

The workflow followed for the database development is depicted in Figure S-4. Extracted Ion Chromatogram (EIC) was exported for each compound setting the accurate $\mathrm{m} / \mathrm{z}$ as calculated from IsotopePattern application tool (Bruker Daltonics Software). Subsequently, Extracted Ion Mobilogram (EIM) was exported given the particular $\mathrm{m} / \mathrm{z}$ and setting retention time limitations according to the elution range of the compound. Finally, MS/MS spectrum was acquired through PASEF mode, taking into account m/z, retention time and mobility range. The first 2 fragments, prioritized by intensity (or in some cases of high intensity, 3 fragments) were included in the database. It is also worth mentioning that the fragments recorded in the database address to a very specific compound's configuration, eliminating the possibility of matching to potential isomers of the compound that may differ in terms of fragmentation as well.

As a result, each compound included in the database is uniquely characterized in terms of $\mathrm{m} / \mathrm{z}$, retention time, ion mobility and fragmentation ions, thus declaring database's significance towards high-confidence identification.

(A)
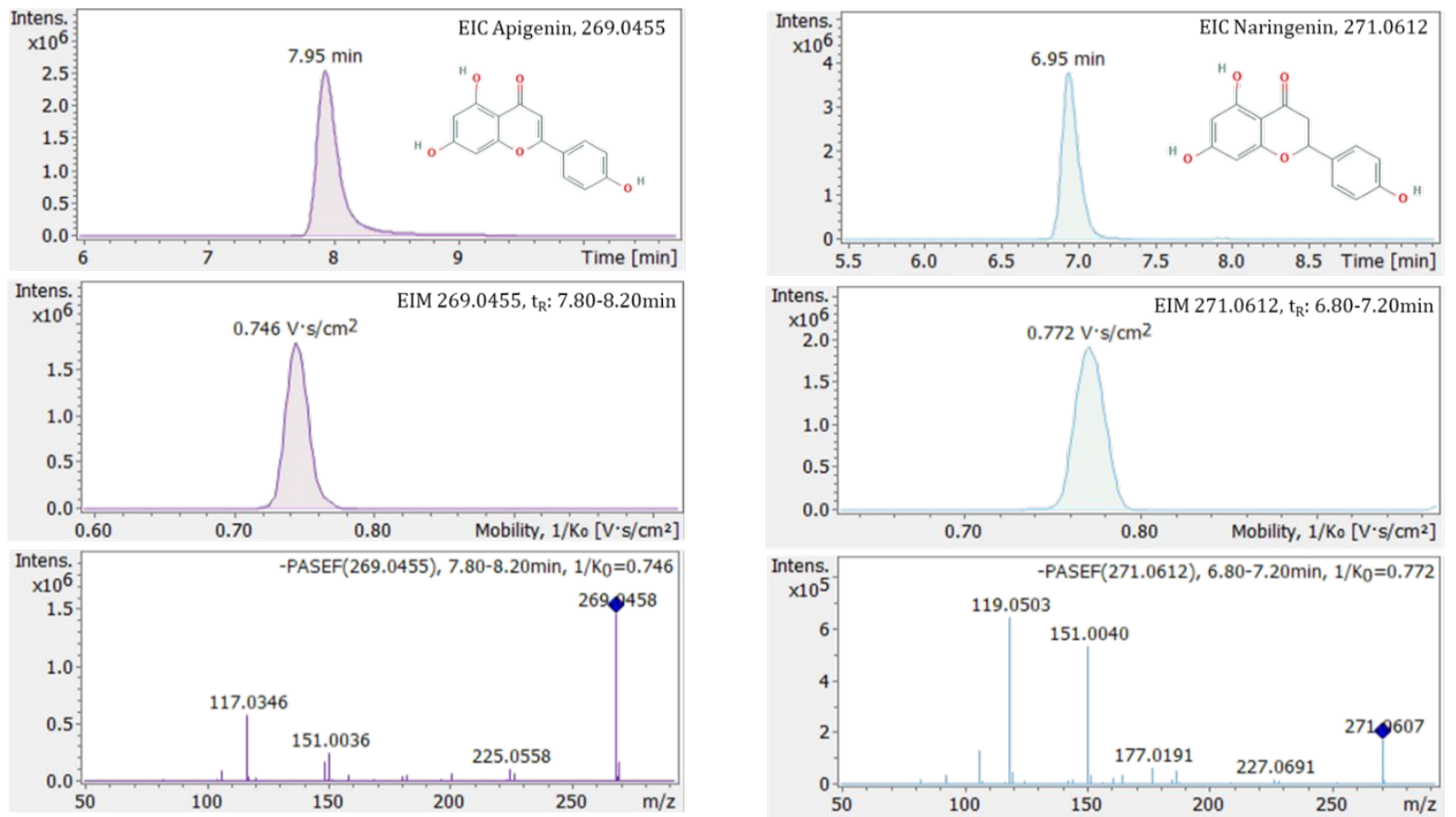

Figure S-5. Representative examples of Apigenin and Naringenin for the acquisition of chromatographic, mobility and spectral information, used in target database development. Extracted Ion Chromatography (EIC) (A) and Extracted Ion Mobilogram (EIM) (B) of each compound were exported, as well as their MS/MS spectra (C). 
Table S-1. EVOOs CCS-enriched target database.

\begin{tabular}{|c|c|c|c|c|c|c|}
\hline Compound & $\begin{array}{c}\text { Molecular } \\
\text { Formula }\end{array}$ & Ions & $\begin{array}{c}\mathbf{t}_{\mathrm{R}} \\
(\mathrm{min})\end{array}$ & $\operatorname{CCS}\left(\mathbf{A}^{2}\right)^{*}$ & $\begin{array}{c}\text { Fragment } \\
\mathbf{m} / \mathbf{z}\end{array}$ & $\begin{array}{c}\text { Elemental } \\
\text { Formula }\end{array}$ \\
\hline Apigenin & $\mathrm{C}_{15} \mathrm{H}_{10} \mathrm{O}_{5}$ & {$[\mathrm{M}-\mathrm{H}]^{-},[\mathrm{M}+\mathrm{H}]^{+}$} & 7.95 & 157.4 & $\begin{array}{l}117.0346 \\
151.0037\end{array}$ & $\begin{array}{l}\mathrm{C}_{8} \mathrm{H}_{5} \mathrm{O}^{1-} \\
\mathrm{C}_{7} \mathrm{H}_{3} \mathrm{O}_{4}{ }^{1-}\end{array}$ \\
\hline Caffeic acid & $\mathrm{C}_{9} \mathrm{H}_{8} \mathrm{O}_{4}$ & {$[\mathrm{M}-\mathrm{H}]^{-}$} & 2.96 & 130.9 & 135.0452 & $\mathrm{C}_{8} \mathrm{H}_{7} \mathrm{O}_{2}{ }^{1-}$ \\
\hline Catechin & $\mathrm{C}_{15} \mathrm{H}_{14} \mathrm{O}_{6}$ & {$[\mathrm{M}-\mathrm{H}]^{-},[\mathrm{M}+\mathrm{H}]^{+}$} & 4.08 & 156.7 & $\begin{array}{l}123.0452 \\
203.0714 \\
151.0401\end{array}$ & $\begin{array}{c}\mathrm{C}_{7} \mathrm{H}_{7} \mathrm{O}_{2}{ }^{1-} \\
\mathrm{C}_{12} \mathrm{H}_{11} \mathrm{O}_{3}{ }^{1-} \\
\mathrm{C}_{8} \mathrm{H}_{7} \mathrm{O}_{3}{ }^{1-}\end{array}$ \\
\hline Epicatechin & $\mathrm{C}_{15} \mathrm{H}_{14} \mathrm{O}_{6}$ & $\begin{array}{c}{[\mathrm{M}-\mathrm{H}]^{-},[\mathrm{M}+\mathrm{H}]^{+}} \\
{[\mathrm{M}+\mathrm{NH} 4]^{+}}\end{array}$ & 4.47 & 156.8 & 123.0452 & $\mathrm{C}_{7} \mathrm{H}_{7} \mathrm{O}_{2}{ }^{1-}$ \\
\hline & & & & & $\begin{array}{l}151.0401 \\
203.0714\end{array}$ & $\begin{array}{c}\mathrm{C}_{8} \mathrm{H}_{7} \mathrm{O}_{3}{ }^{1-} \\
\mathrm{C}_{12} \mathrm{H}_{11} \mathrm{O}_{3}{ }^{1-}\end{array}$ \\
\hline Eriodictyol & $\mathrm{C}_{15} \mathrm{H}_{12} \mathrm{O}_{6}$ & {$[\mathrm{M}-\mathrm{H}]^{-},[\mathrm{M}+\mathrm{H}]^{+}$} & 6.37 & 164.5 & $\begin{array}{l}135.0452 \\
151.0037 \\
136.0530\end{array}$ & $\begin{array}{l}\mathrm{C}_{8} \mathrm{H}_{7} \mathrm{O}_{2}{ }^{1-} \\
\mathrm{C}_{7} \mathrm{H}_{3} \mathrm{O}_{4}{ }^{1-} \\
\mathrm{C}_{8} \mathrm{H}_{8} \mathrm{O}_{2}{ }^{1-}\end{array}$ \\
\hline Ferulic acid & $\mathrm{C}_{10} \mathrm{H}_{10} \mathrm{O}_{4}$ & {$[\mathrm{M}-\mathrm{H}]^{-},[\mathrm{M}+\mathrm{H}]^{+}$} & 3.55 & 137.8 & $\begin{array}{l}134.0373 \\
178.0272\end{array}$ & $\begin{array}{l}\mathrm{C}_{8} \mathrm{H}_{6} \mathrm{O}_{2}{ }^{1-} \\
\mathrm{C}_{9} \mathrm{H}_{6} \mathrm{O}_{4}{ }^{1-}\end{array}$ \\
\hline Kaempferol & $\mathrm{C}_{15} \mathrm{H}_{10} \mathrm{O}_{6}$ & $\begin{array}{c}{[\mathrm{M}-\mathrm{H}]^{-},[\mathrm{M}+\mathrm{H}]^{+}} \\
{[\mathrm{M}+\mathrm{Na}]^{+}}\end{array}$ & 8.03 & 261.0 & 185.0608 & $\mathrm{C}_{12} \mathrm{H}_{9} \mathrm{O}_{2}{ }^{1-}$ \\
\hline Ligstroside aglycone & $\mathrm{C}_{19} \mathrm{H}_{22} \mathrm{O}_{7}$ & {$[\mathrm{M}-\mathrm{H}]^{-},[\mathrm{M}+\mathrm{H}]^{+}$} & 8.03 & $274.0^{*}$ & $\begin{array}{l}229.0506 \\
291.0874 \\
127.0401 \\
101.0244\end{array}$ & $\begin{array}{c}\mathrm{C}_{13} \mathrm{H}_{9} \mathrm{O}_{4}{ }^{1-} \\
\mathrm{C}_{15} \mathrm{H}_{15} \mathrm{O}_{6}{ }^{1-} \\
\mathrm{C}_{6} \mathrm{H}_{7} \mathrm{O}_{3}{ }^{1-} \\
\mathrm{C}_{4} \mathrm{H}_{5} \mathrm{O}_{3}{ }^{1-}\end{array}$ \\
\hline Luteolin & $\mathrm{C}_{15} \mathrm{H}_{10} \mathrm{O}_{6}$ & $\begin{array}{c}{[\mathrm{M}-\mathrm{H}]^{-},[\mathrm{M}+\mathrm{H}]^{+}} \\
{[\mathrm{M}+\mathrm{Na}]^{+}}\end{array}$ & 7.82 & 159.9 & 133.0295 & $\mathrm{C}_{8} \mathrm{H}_{5} \mathrm{O}_{2}{ }^{1-}$ \\
\hline Myricetin & $\mathrm{C}_{15} \mathrm{H}_{10} \mathrm{O}_{8}$ & {$[\mathrm{M}-\mathrm{H}]^{-},[\mathrm{M}+\mathrm{H}]^{+}$} & 7.10 & 166.2 & $\begin{array}{l}151.0037 \\
178.9986 \\
137.0244\end{array}$ & $\begin{array}{l}\mathrm{C}_{7} \mathrm{H}_{3} \mathrm{O}_{4}{ }^{1-} \\
\mathrm{C}_{8} \mathrm{H}_{3} \mathrm{O}_{5}{ }^{1-} \\
\mathrm{C}_{7} \mathrm{H}_{5} \mathrm{O}_{3}{ }^{1-}\end{array}$ \\
\hline Naringenin & $\mathrm{C}_{15} \mathrm{H}_{12} \mathrm{O}_{5}$ & {$[\mathrm{M}-\mathrm{H}]^{-},[\mathrm{M}+\mathrm{H}]^{+}$} & 6.95 & 162.4 & $\begin{array}{l}119.0502 \\
151.0037\end{array}$ & $\begin{array}{l}\mathrm{C}_{8} \mathrm{H}_{7} \mathrm{O}^{1-} \\
\mathrm{C}_{7} \mathrm{H}_{3} \mathrm{O}_{4}{ }^{1-}\end{array}$ \\
\hline Oleacein & $\mathrm{C}_{17} \mathrm{H}_{20} \mathrm{O}_{6}$ & {$[\mathrm{M}-\mathrm{H}]^{-},[\mathrm{M}+\mathrm{H}]^{+}$} & 5.88 & 251.3 & $\begin{array}{l}183.0662 \\
195.0662\end{array}$ & $\begin{array}{l}\mathrm{C}_{9} \mathrm{H}_{11} \mathrm{O}_{4}{ }^{1-} \\
\mathrm{C}_{10} \mathrm{H}_{11} \mathrm{O}_{4}{ }^{1-}\end{array}$ \\
\hline Oleocanthal & $\mathrm{C}_{17} \mathrm{H}_{20} \mathrm{O}_{5}$ & {$[\mathrm{M}-\mathrm{H}]^{-},[\mathrm{M}+\mathrm{H}]^{+}$} & 6.51 & 249.7 & 137.0608 & $\mathrm{C}_{8} \mathrm{H}_{9} \mathrm{O}_{2}{ }^{1-}$ \\
\hline Oleocanthalic acid & $\mathrm{C}_{17} \mathrm{H}_{20} \mathrm{O}_{6}$ & {$[\mathrm{M}-\mathrm{H}]^{-},[\mathrm{M}+\mathrm{H}]^{+}$} & 5.22 & $245.8^{*}$ & $\begin{array}{l}199.0612 \\
111.0088\end{array}$ & $\begin{array}{c}\mathrm{C}_{9} \mathrm{H}_{11} \mathrm{O}_{5}{ }^{1-}- \\
\mathrm{C}_{5} \mathrm{H}_{3} \mathrm{O}_{3}{ }^{1-}\end{array}$ \\
\hline Oleokoronal & $\mathrm{C}_{19} \mathrm{H}_{22} \mathrm{O}_{7}$ & {$[\mathrm{M}-\mathrm{H}]^{-},[\mathrm{M}+\mathrm{H}]^{+}$} & 6.68 & 178.8 & $\begin{array}{l}291.0874 \\
127.0401 \\
101.0244\end{array}$ & $\begin{array}{c}\mathrm{C}_{15} \mathrm{H}_{15} \mathrm{O}_{6}{ }^{1-} \\
\mathrm{C}_{6} \mathrm{H}_{7} \mathrm{O}_{3}{ }^{1-} \\
\mathrm{C}_{4} \mathrm{H}_{5} \mathrm{O}_{3}{ }^{1-}\end{array}$ \\
\hline Oleomissional & $\mathrm{C}_{19} \mathrm{H}_{22} \mathrm{O}_{8}$ & {$[\mathrm{M}-\mathrm{H}]^{-},[\mathrm{M}+\mathrm{H}]^{+}$} & 6.07 & 184.7 & $\begin{array}{l}111.0088 \\
139.0037 \\
149.0244\end{array}$ & $\begin{array}{l}\mathrm{C}_{5} \mathrm{H}_{3} \mathrm{O}_{3}{ }^{1-} \\
\mathrm{C}_{6} \mathrm{H}_{3} \mathrm{O}_{4}{ }^{1-} \\
\mathrm{C}_{8} \mathrm{H}_{5} \mathrm{O}_{3}{ }^{1-}\end{array}$ \\
\hline Oleuropein & $\mathrm{C}_{25} \mathrm{H}_{32} \mathrm{O}_{13}$ & $\begin{array}{c}{[\mathrm{M}-\mathrm{H}]^{-},[\mathrm{M}+\mathrm{NH} 4]^{+}} \\
{[\mathrm{M}+\mathrm{H}]^{+}}\end{array}$ & 5.98 & 217.6 & 307.0823 & $\mathrm{C}_{15} \mathrm{H}_{15} \mathrm{O}_{7}^{1-}$ \\
\hline Oleuropein aglycone & $\mathrm{C}_{19} \mathrm{H}_{22} \mathrm{O}_{8}$ & {$[\mathrm{M}-\mathrm{H}]^{-},[\mathrm{M}+\mathrm{H}]^{+}$} & 7.59 & $270.0^{*}$ & $\begin{array}{l}275.0925 \\
111.0088 \\
139.0037 \\
149.0244\end{array}$ & $\begin{array}{c}\mathrm{C}_{15} \mathrm{H}_{15} \mathrm{O}_{5}{ }^{1-} \\
\mathrm{C}_{5} \mathrm{H}_{3} \mathrm{O}_{3}{ }^{1-} \\
\mathrm{C}_{6} \mathrm{H}_{3} \mathrm{O}_{4}{ }^{1-} \\
\mathrm{C}_{8} \mathrm{H}_{5} \mathrm{O}_{3}{ }^{1-}\end{array}$ \\
\hline Phlorizin & $\mathrm{C}_{21} \mathrm{H}_{24} \mathrm{O}_{10}$ & {$[\mathrm{M}-\mathrm{H}]^{-},[\mathrm{M}+\mathrm{H}]^{+}$} & 5.69 & 195.1 & $\begin{array}{l}273.0768 \\
167.0350\end{array}$ & $\begin{array}{c}\mathrm{C}_{15} \mathrm{H}_{13} \mathrm{O}_{5}{ }^{1-} \\
\mathrm{C}_{8} \mathrm{H}_{7} \mathrm{O}_{4}{ }^{1-}\end{array}$ \\
\hline Pinoresinol & $\mathrm{C}_{20} \mathrm{H}_{22} \mathrm{O}_{6}$ & {$[\mathrm{M}-\mathrm{H}]^{-},[\mathrm{M}+\mathrm{H}]^{+}$} & 6.48 & 196.7 & 151.0401 & $\mathrm{C}_{8} \mathrm{H}_{7} \mathrm{O}_{3}{ }^{1-}$ \\
\hline Quercetin & $\mathrm{C}_{15} \mathrm{H}_{10} \mathrm{O}_{7}$ & {$[\mathrm{M}-\mathrm{H}]^{-},[\mathrm{M}+\mathrm{H}]^{+}$} & 7.40 & 163.1 & $\begin{array}{l}151.0037 \\
178.9986\end{array}$ & $\begin{array}{l}\mathrm{C}_{7} \mathrm{H}_{3} \mathrm{O}_{4}{ }^{1-} \\
\mathrm{C}_{8} \mathrm{H}_{3} \mathrm{O}_{5}{ }^{1-}\end{array}$ \\
\hline Vanillin & $\mathrm{C}_{8} \mathrm{H}_{8} \mathrm{O}_{3}$ & {$[\mathrm{M}-\mathrm{H}]^{-},[\mathrm{M}+\mathrm{H}]^{+}$} & 6.48 & 206.7 & $\begin{array}{l}136.0166 \\
108.0217 \\
\end{array}$ & $\begin{array}{l}\mathrm{C}_{7} \mathrm{H}_{4} \mathrm{O}_{3}{ }^{1-} \\
\mathrm{C}_{6} \mathrm{H}_{4} \mathrm{O}_{2}{ }^{1-} \\
\end{array}$ \\
\hline
\end{tabular}

\footnotetext{
${ }^{*}$ CCS of major conformer.
} 
CCS values were evaluated regarding their robustness through $\triangle \mathrm{CCS}$ estimation, calculated according to the following equation: ${ }^{4}$

$$
\% \Delta \mathrm{CCS}=\frac{\text { CCS }^{\text {measured }}-\text { CCS }^{\text {database }}}{C C S^{\text {database }}} \times 100
$$

Table S-2 summarizes the CCS values exported from each ionization run, in both negative and positive. $\% \Delta C C S$ is calculated for each compound detected in both acquisition modes, as well as an absolute average value.

Table S-2. \%CCS error as calculated comparing negative and positive acquisition mode.

\begin{tabular}{|c|c|c|c|}
\hline Compound & $\begin{array}{c}C C S\left(\AA^{2}\right) \\
\text { Negative mode }\end{array}$ & $\begin{array}{c}\operatorname{CCS}\left(\AA^{2}\right) \\
\text { Positive mode }\end{array}$ & $\% \Delta \mathrm{CCS}$ \\
\hline Apigenin & 157.4 & 159.9 & 1.57 \\
\hline Caffeic acid & 130.9 & 130.4 & -0.41 \\
\hline Catechin & 156.7 & 162.7 & 3.85 \\
\hline Epicatechin & 156.8 & 162.7 & 3.76 \\
\hline Eriodictyol & 164.5 & 166.9 & 1.46 \\
\hline Ferulic acid & 137.8 & 137.1 & -0.53 \\
\hline Kaempferol & 161.0 & 164.7 & 2.31 \\
\hline Ligstroside aglycone & 274.0 & 271.8 & -0.78 \\
\hline Luteolin & 159.9 & 164.3 & 2.73 \\
\hline Myricetin & 166.2 & 171.3 & 3.05 \\
\hline Naringenin & 162.4 & 163.6 & 0.75 \\
\hline Oleacein & 251.3 & 252.4 & 0.44 \\
\hline Oleocanthal & 249.7 & 250.7 & 0.39 \\
\hline Oleocanthalic acid & 245.8 & 253.5 & 3.12 \\
\hline Oleokoronal & 178.8 & 180.4 & 0.89 \\
\hline Oleomissional & 184.7 & 191.5 & 3.70 \\
\hline Oleuropein & 217.6 & 218.1 & 0.23 \\
\hline Oleuropein aglycone & 270.0 & 270.0 & -0.02 \\
\hline Phlorizin & 195.1 & 191.3 & -1.96 \\
\hline Pinoresinol & 196.7 & 189.6 & -3.61 \\
\hline Quercetin & 163.1 & 167.2 & 2.54 \\
\hline Vanillin & 206.7 & 202.6 & -2.00 \\
\hline $\begin{array}{c}\text { Average } \\
\text { (absolute bias) }\end{array}$ & & & 1.82 \\
\hline
\end{tabular}


Table S-3 summarizes the CCS values exported in other IMS studies using TIMS, same as in our study, ${ }^{5}$ and Drift Tube IMS (DTIMS). ${ }^{6}$ An absolute average value of $\% \triangle \mathrm{CCS}$ in each study is also calculated.

Table S-3. $\triangle$ CCS as calculated comparing CCS exported in other IMS studies.

\begin{tabular}{|c|c|c|c|c|c|}
\hline Compound & $\operatorname{CCS}\left(\AA^{2}\right)$ & $\operatorname{CCS}\left(\AA^{2}\right)$ TIMS $^{5}$ & $\%$ CCS error & $\operatorname{CCS}\left(\AA^{2}\right)$ DTIMS $^{6}$ & $\% \Delta C C S$ \\
\hline Apigenin & 157.4 & 157.1 & 0.21 & - & - \\
\hline Catechin & 156.7 & 157.0 & -0.21 & 158.1 & -0.91 \\
\hline Epicatechin & 156.8 & - & - & 158.4 & -1.01 \\
\hline Eriodictyol & 164.5 & 165.5 & -0.60 & - & - \\
\hline Ferulic acid & 137.8 & - & - & 139.7 & -1.37 \\
\hline Luteolin & 159.9 & 160.2 & -0.22 & - & - \\
\hline Myricetin & 166.2 & - & & 168.4 & -1.31 \\
\hline Naringenin & 162.4 & 162.6 & -0.11 & 164.9 & -1.53 \\
\hline Quercetin & 163.1 & - & - & 165.5 & -1.46 \\
\hline $\begin{array}{c}\text { Average } \\
\text { (absolute bias) }\end{array}$ & & & 0.27 & & 1.26 \\
\hline
\end{tabular}

Table S-4 summarizes the CCS values exported in standard solution analysis compared to values recorded in EVOOs samples in order to evaluate CCS matrix effect. An absolute average value of $\% \Delta \mathrm{CCS}$ is also calculated. Despite the fact that more than one mobility peaks were detected in EVOOs samples, especially in the case of secoiridoids compounds, however only mobility peaks also detected in standard's are included for direct-comparison reasons.

Table S-4. $\% \Delta C C S$ as calculated evaluating CCS matrix effect.

\begin{tabular}{c|ccc}
\hline Compound & $\begin{array}{c}\text { CCS }\left(\AA^{\mathbf{2}}\right) \\
\text { Standard analysis }\end{array}$ & $\begin{array}{c}\text { CCS }\left(\AA^{\mathbf{2}}\right) \\
\text { EVOOs analysis }\end{array}$ & $\% \Delta \mathbf{C C S}$ \\
\hline Apigenin & 157.4 & 157.4 & 0.00 \\
Caffeic acid & 130.9 & $\mathrm{ND}$ & - \\
Catechin & 156.7 & $\mathrm{ND}$ & - \\
Epicatechin & 156.8 & $\mathrm{ND}$ & - \\
Eriodictyol & 164.5 & 164.7 & - \\
Ferulic acid & 137.8 & $\mathrm{ND}$ & - \\
Kaempferol & 161.0 & $\mathrm{ND}$ & -1.06 \\
Ligstroside aglycone & 274.0 & $271.1^{*}$ & 0.27 \\
Luteolin & 159.9 & 160.3 & - \\
Myricetin & 166.2 & $\mathrm{ND}$ & 0.39 \\
Naringenin & 162.4 & 163.0 & 0.06 \\
Oleacein & 251.3 & 251.5 & 0.32 \\
Oleocanthal & 249.7 & 250.5 & \\
& & &
\end{tabular}




\begin{tabular}{c|ccc} 
Oleocanthalic acid & 245.8 & $247.7^{*}$ & 0.76 \\
Oleokoronal & 178.8 & $178.8^{*}$ & -0.02 \\
Oleomissional & 184.7 & $184.9^{*}$ & 0.12 \\
Oleuropein & 217.6 & $\mathrm{ND}$ & - \\
Oleuropein aglycone & 270.0 & $270.6^{*}$ & 0.21 \\
Phlorizin & 195.1 & $\mathrm{ND}$ & - \\
Pinoresinol & 196.7 & 197.6 & 0.42 \\
Quercetin & 163.1 & $\mathrm{ND}$ & - \\
Vanillin & 206.7 & 207.3 & 0.32 \\
\hline $\begin{array}{c}\text { Average } \\
\text { (absolute bias) }\end{array}$ & & & 0.31
\end{tabular}

${ }^{*} \mathrm{CCS}$ detected in standard's solution as well. ND: not detected 
(A)

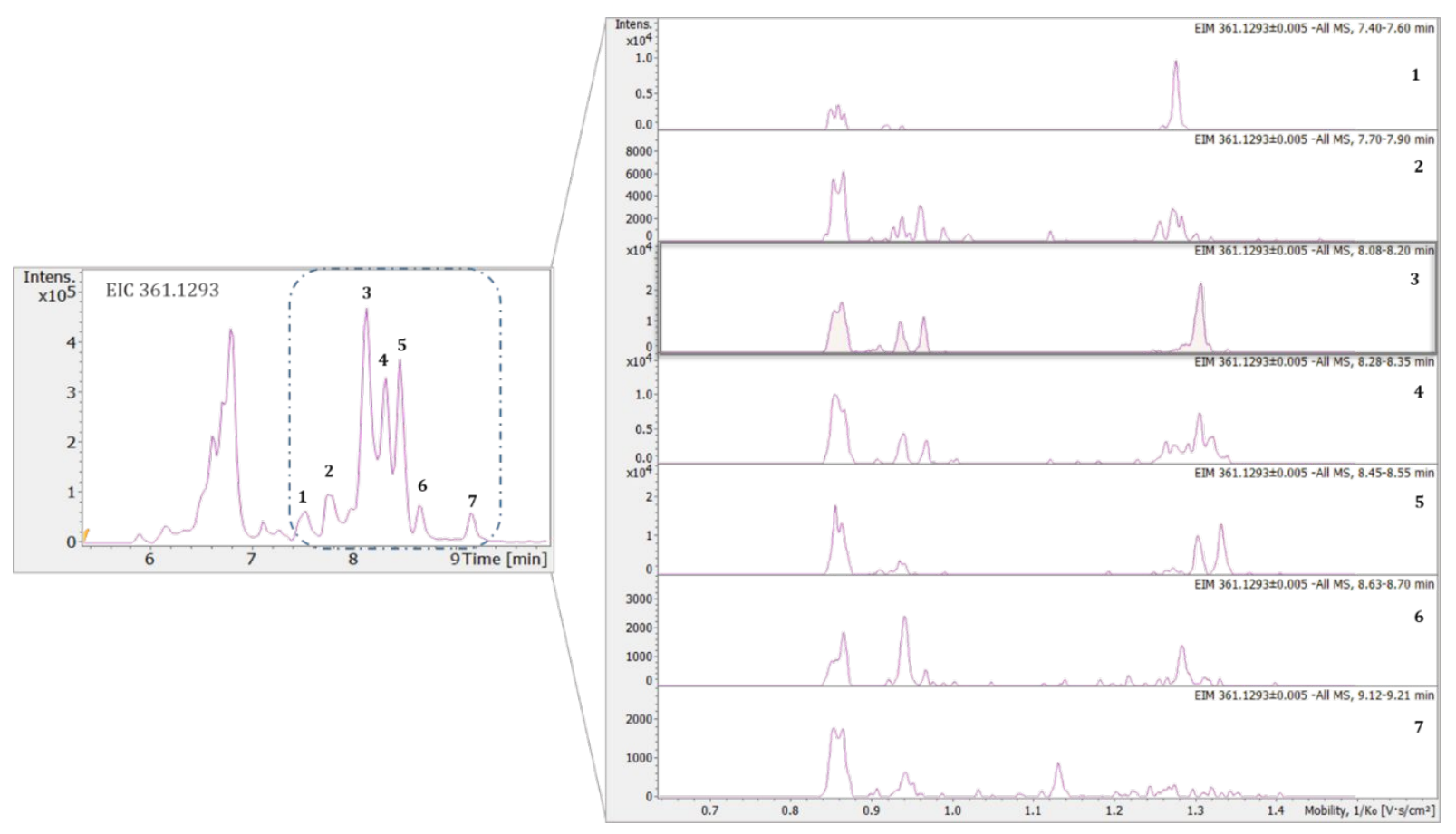

(B)
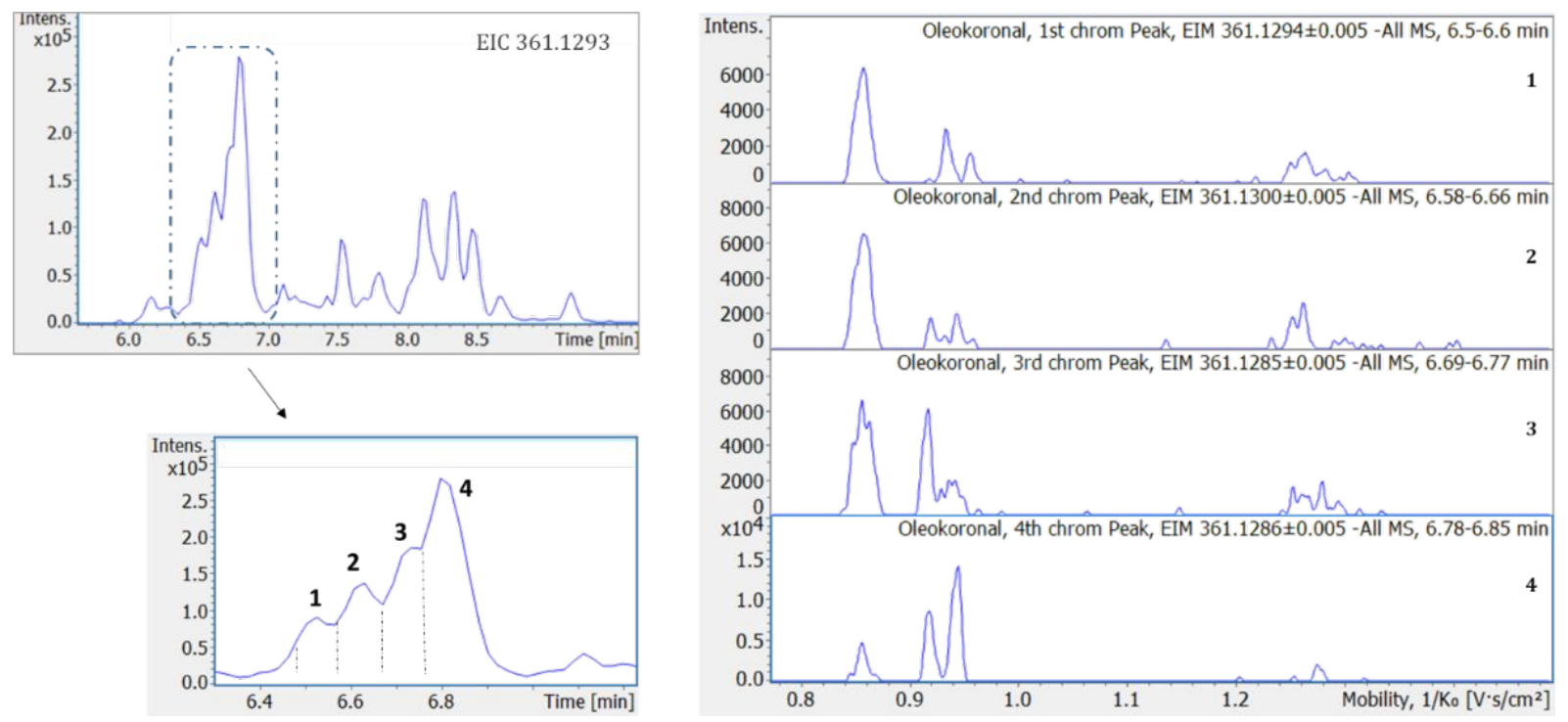

Figure S-6. Extracted Ion Mobility (EIM) for $361.1293 \mathrm{~m} / \mathrm{z}$. Setting retention time filtering limitations ligstroside aglycone presents 7 chromatographic peaks (A), while Oleokoronal 4 chromatographic peaks respectively (B), Different mobility profile is recorded for each respective chromatographic peak, in terms of intensity as well. 


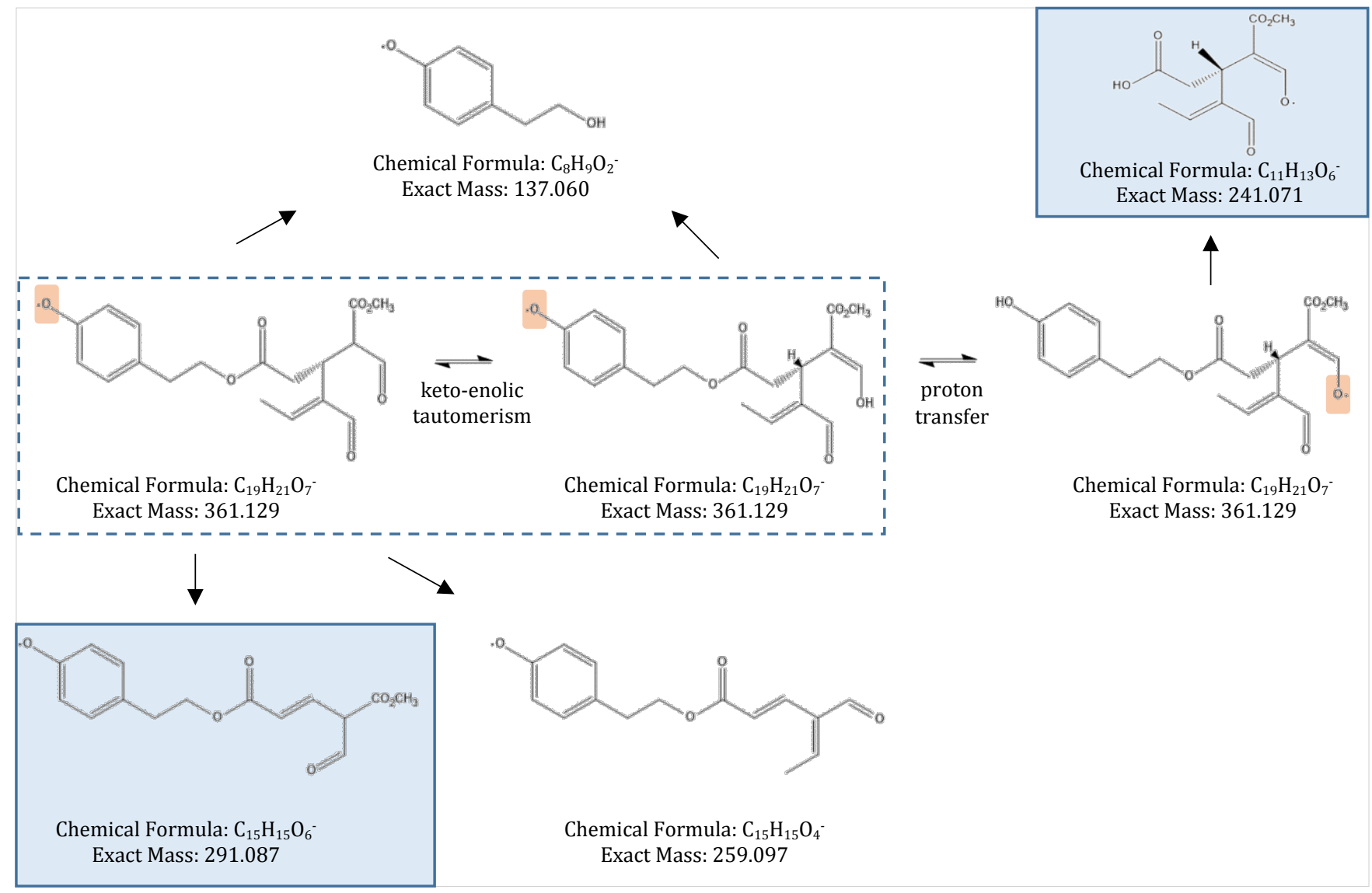

Figure S-7. Fragmentation pathways to explain $\mathrm{m} / \mathrm{z}$ detected in Ligstroside aglycone open form MS/MS spectra, in negative acquisition mode. ${ }^{7}$ Different fragments are detected depending on the part of the compound that deprotonation occurs. When deprotonation takes place at the side of the benzene ring, the main fragment detected is the 291.087 ion. On the contrary, when the compound is deprotonated at the other side of the molecule, 241.071 is produced as the main fragment. 


\section{SI-7 Holistic untargeted data treatment workflow}

A Spectral Library (SL) comprised of more than $50 \mathrm{MS} / \mathrm{MS}$ spectra of natural products was used for features annotation. The development of a SL contains information regarding compound's name, formula, retention time recorded, precursor's exact mass, polarity detected and collision energy applied in fragmentation process. Furthermore, mass accuracy is (expressed in $\mathrm{mDa}$ ) can be adjusted according to each study acceptable tolerance. Figure S-9 illustrates SL's contribution to discriminate and successfully annotate 3 compounds (Apigenin, Galangin and Genistein) of similar structure.

All 3 compounds have the same formula $\left(\mathrm{C}_{15} \mathrm{H}_{10} \mathrm{O}_{5}\right)$, but they differ in structure arrangement, with the hydroxyl group being set in different part in the benzene ring. In MS/MS Library Search tab, as visualized in MetaboScape environment and in the following figures, standard's spectrum, already registered in the library, is highlighted under the "Spectral Library" label, while "Query" corresponds to the feature under investigation. From spectra research for all 3 spectra compounds, we conclude that Apigenin is the compound best fitting in the MS/MS profile, and thus is further annotated under Apigenin name.

(A)

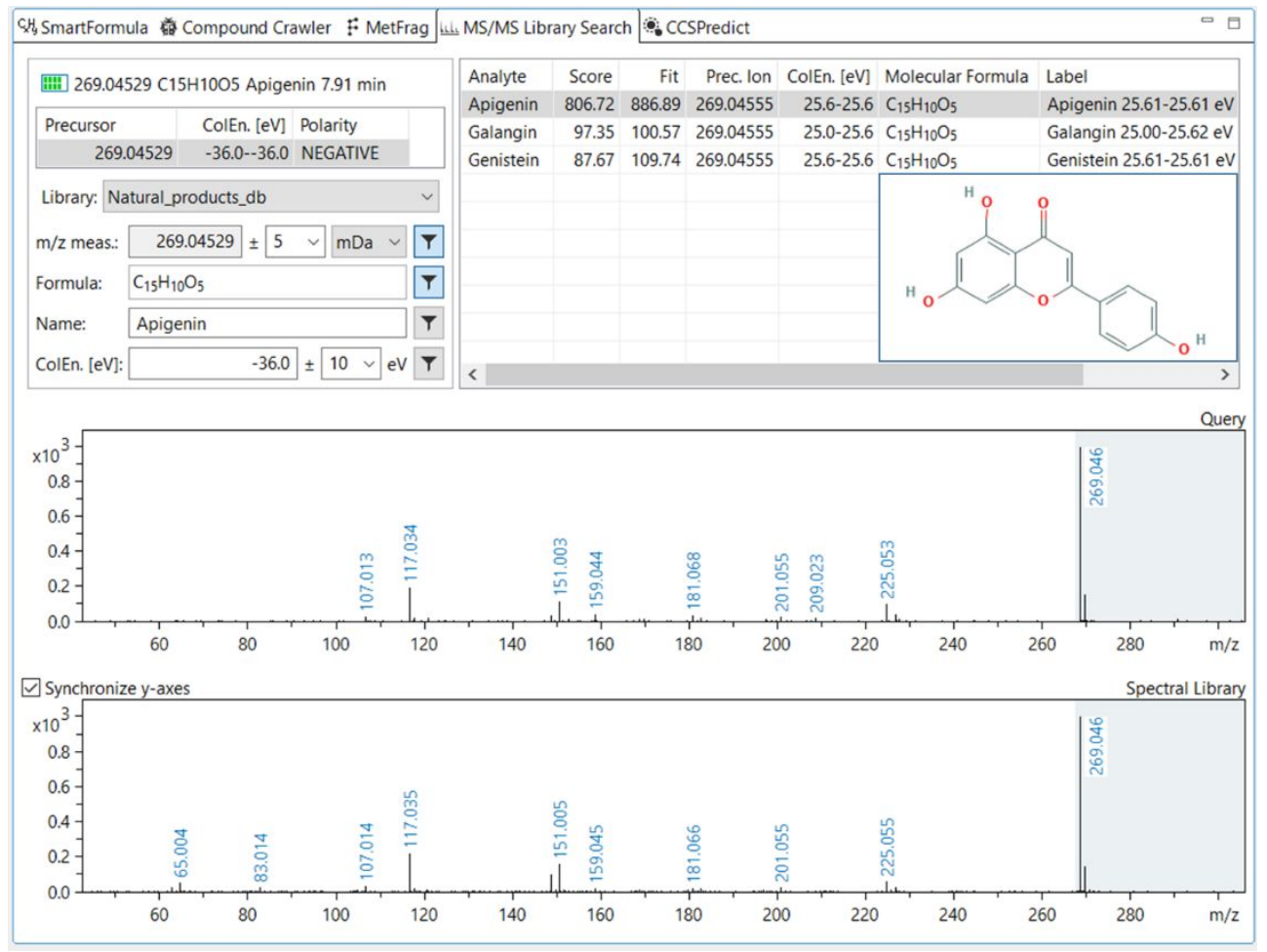


(B)

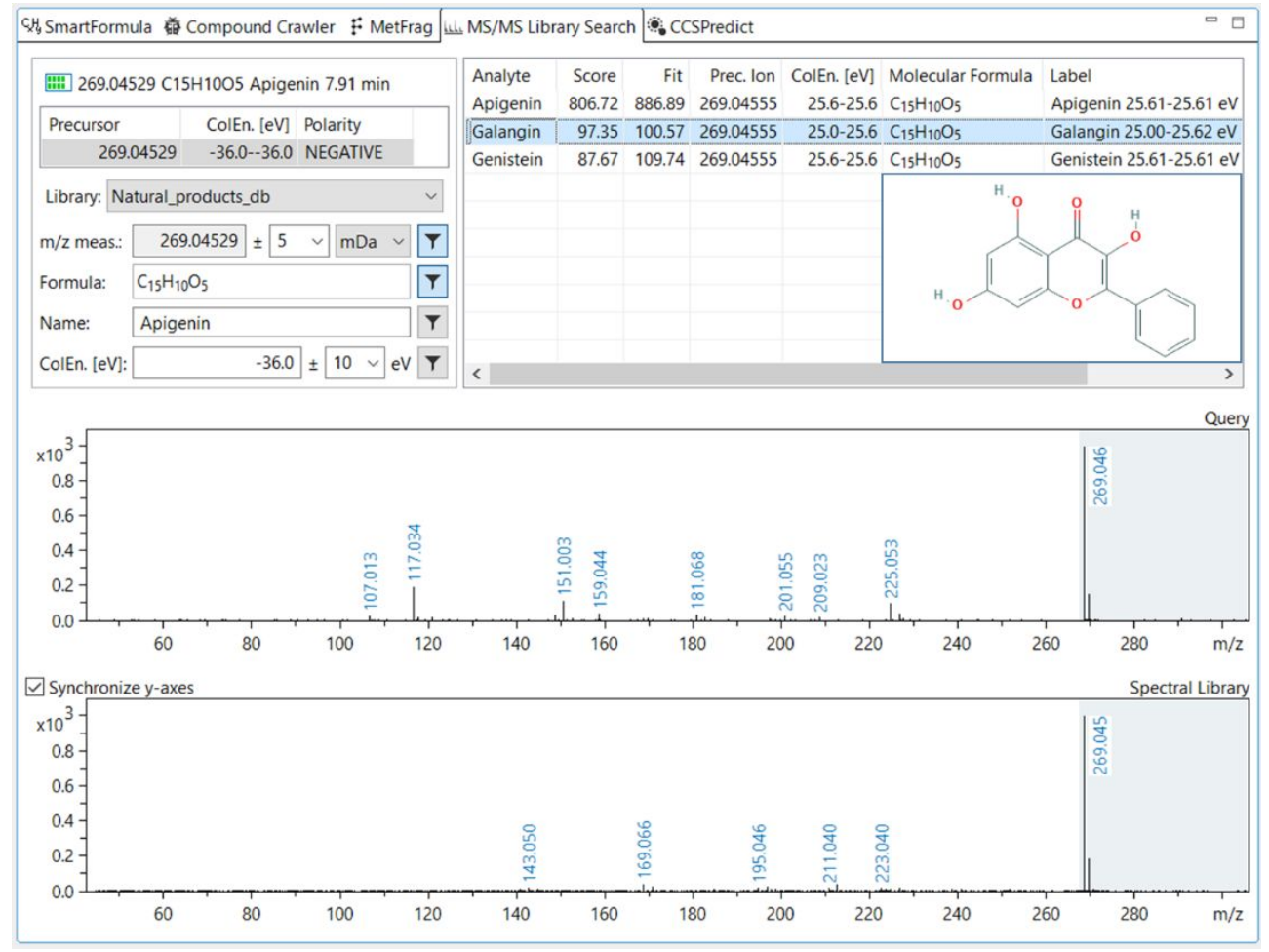

(C)

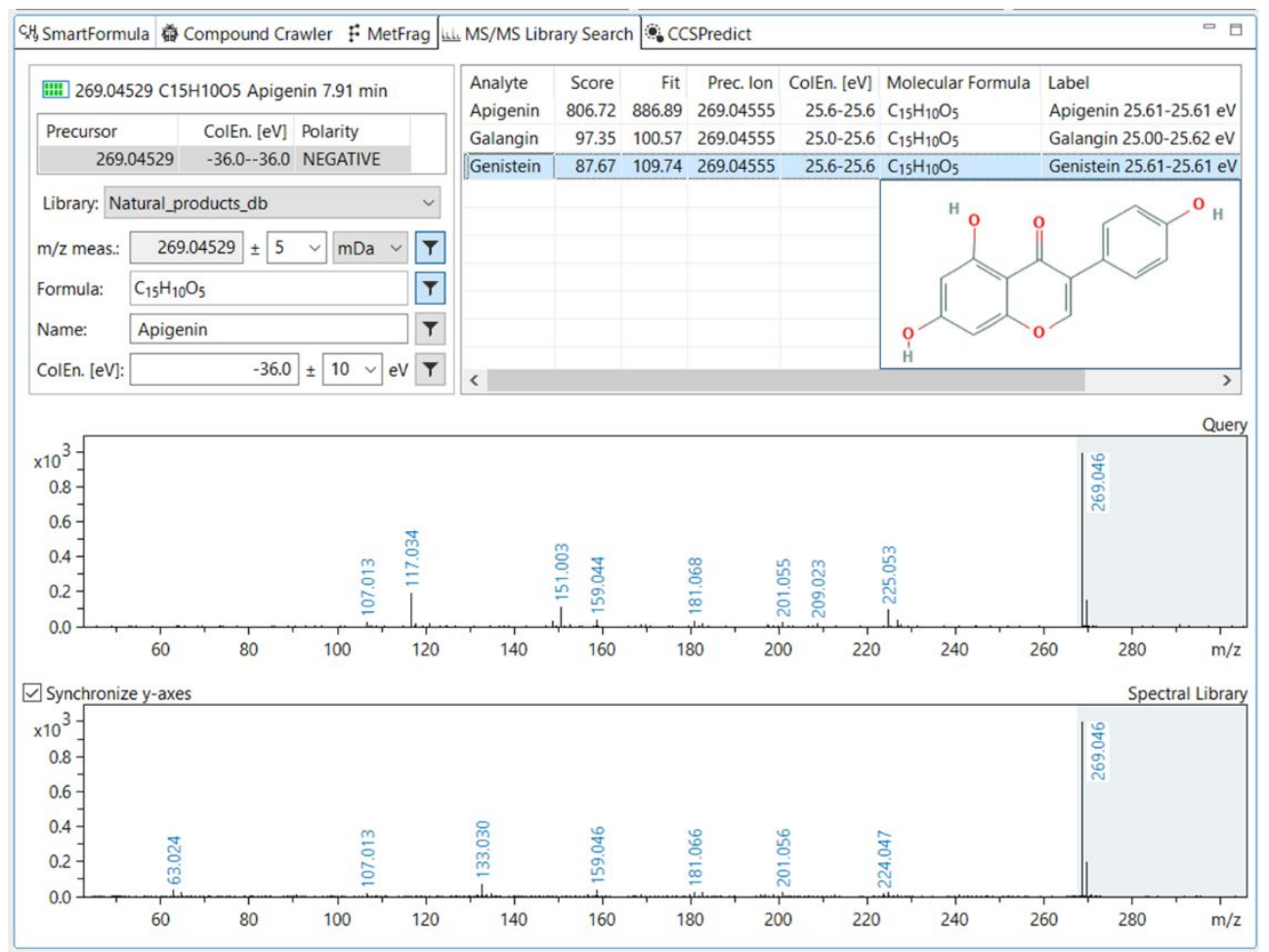

Figure S-8. Spectral library environment in MetaboScape software. MS/MS spectrum of the compound under identification (notified as "Query spectrum") is compared to MS/MS spectra of compounds included in the spectral library. 3 compounds of high structure similarity, Apigenin (A), Galangin (B) and Genistein (C), are presented as identification candidates, with Apigenin spectral profile best matching in the MS/MS profile of the investigated feature. 
Annotation Quality (AQ)

Figure S-10 illustrates a typical chromatographic profile (BPC) as acquired in negative ionization mode (A). Bioactive compounds are mainly detected in the first 12 minutes approximately of the chromatography, with the class of fatty acids following elution gradient, as compounds of lower polarity. The aforementioned observation is declared in the bucket table exported as well, with features annotation based on EVOOs CCS-enriched target database. Identification confidence is expressed through of Annotation Quality (AQ) score. Feature annotation was evaluated making use of MetaboScape embedded tool of AQ score. Features extracted were evaluated for their matching with theoretical values in terms of mass accuracy $(\mathrm{mDa})$, retention time (min), isotopic pattern (mSigma), MS/MS fragmentation and CCS. The matching parameters are user-defined, with a narrow (green colour) and wide (grey colour) range of confidence annotation to be customized in each measured parameter. Regarding CCS identification matching parameter, CCS accuracy of $2 \%$ was set as narrow range of confidence, reported at the 5 -level confidence scheme proposed by Celma et al. ${ }^{8}$

(A)

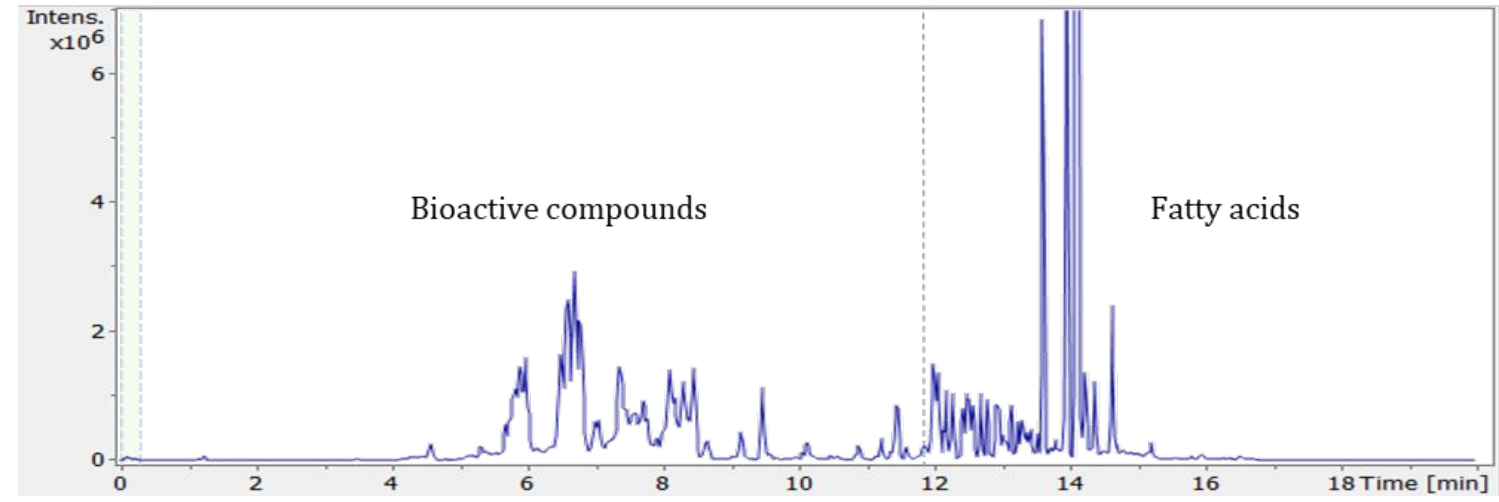

(B)

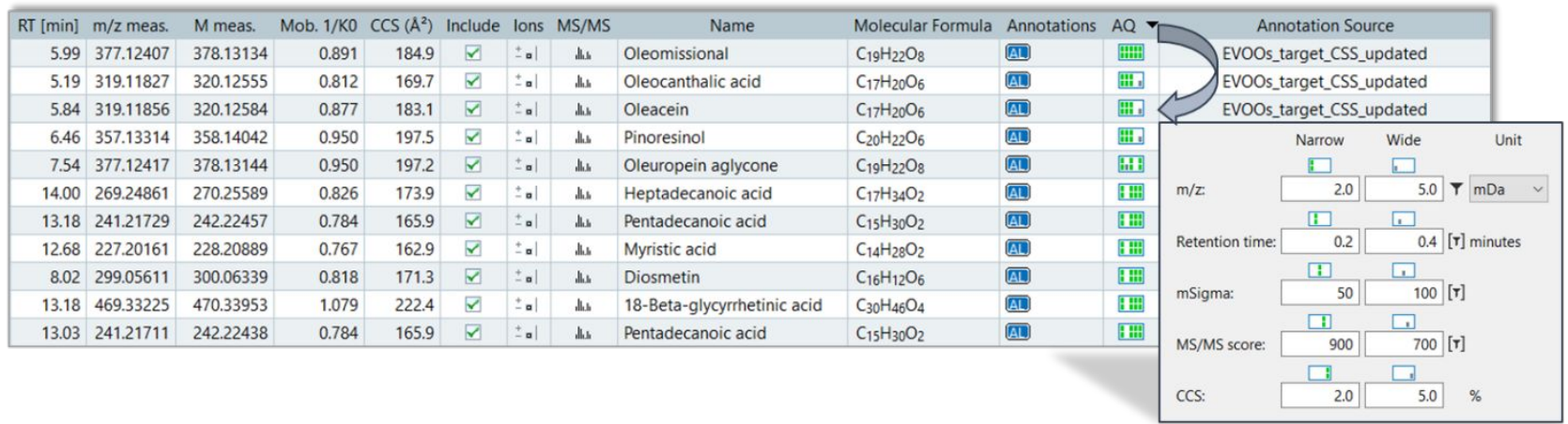

Figure S-9. Typical chromatographic profile (BPC) as acquired in negative ionization mode (A). Bioactive compounds are mainly detected in the first 12 minutes approximately of the chromatography, with the class of fatty acids following elution gradient. Representative report in Bruker MetaboScape software (B). Each feature extracted is annotated using software respective tools (e.g. analyte list) and further evaluated through annotation quality (AQ) score. The annotation quality (AQ) column illustrates the matching confidence in terms of accurate mass, retention time, isotopic pattern (mSigma), MS/MS fragmentation, and CCS (from left to right). The matching parameters are userdefined, with a narrow (green colour) and wide (grey colour) range of confidence annotation to be customized in each measured parameter. 


\section{EVOOs Variety Discrimination}

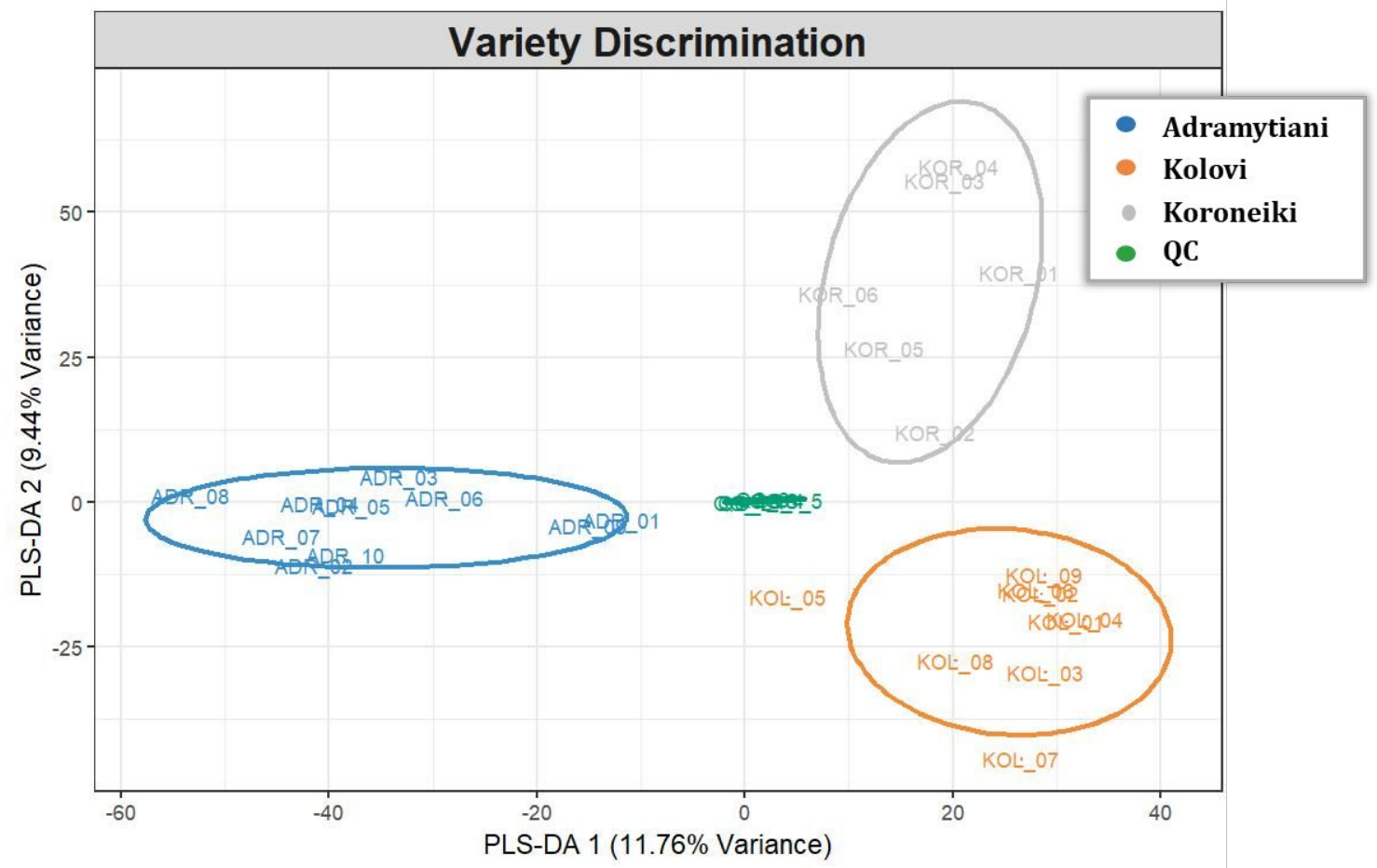

Figure S-10. PLS-DA loading plot bounded by the first two components of the analysis in EVOOs variety discrimination study. All features included in the merged data set have been introduced in the model, resulting to distinct discrimination among all the 3 groups of variety.

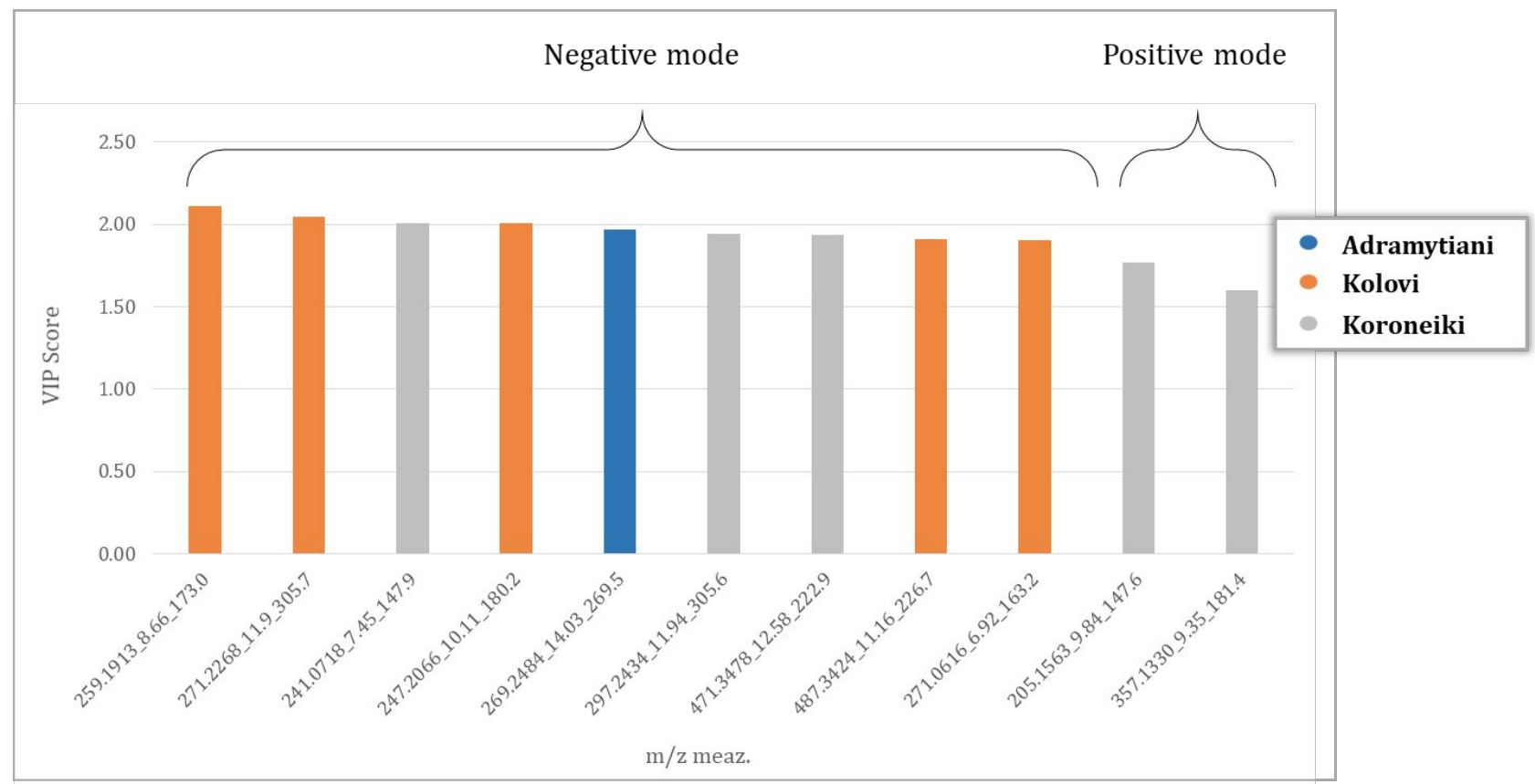

Figure S-11. Most important markers in EVOOs variety discrimination as highlighted by PLS-DA VIP score. In y-axis, features detected are coded according to the format "accurate mass $(\mathrm{m} / \mathrm{z})$ retention time_CCS value", that corresponds to experimental measurements of each feature in particular. Markers of each variety are depicted, labelled at different colours, while their acquisition mode (negative/positive) is also clarified. 
Table S-5. Identified metabolites in EVOOs variety discrimination study based on positive and negative mode LC-TIMS-TOFMS measurements, classified according to their VIP score. Features detected are coded according to the format "accurate mass (m/z)_retention time_CCS value", that corresponds to experimental measurements of each feature in particular.

\begin{tabular}{|c|c|c|c|c|c|c|c|c|c|}
\hline Features & $\begin{array}{c}\mathrm{m} / \mathbf{z} \\
\text { meas. }\end{array}$ & $\begin{array}{c}t_{R} \\
(\min )\end{array}$ & $\begin{array}{l}\text { CCS } \\
\left(\AA^{2}\right)\end{array}$ & Ions & Fragments & $\begin{array}{l}\text { Putative } \\
\text { Formula }\end{array}$ & Identification & $\begin{array}{c}\text { ID } \\
\text { Level }\end{array}$ & Marker \\
\hline 259.1913_8.66_173.0 & 259.1913 & 8.66 & 173.0 & {$[\mathrm{M}-\mathrm{H}]^{-}$} & $\begin{array}{l}101.0609 \\
193.1582 \\
197.1883\end{array}$ & $\mathrm{C}_{14} \mathrm{H}_{28} \mathrm{O}_{4}$ & - & 4 & Kolovi \\
\hline 271.2278_11.90_305.7 & 271.2268 & 11.90 & 305.7 & {$[\mathrm{M}-\mathrm{H}]^{-}$} & 197.1880 & $\mathrm{C}_{16} \mathrm{H}_{32} \mathrm{O}_{3}$ & $\begin{array}{l}\text { 16-Hydroxy-hexa } \\
\text { decanoic acid }{ }^{2}\end{array}$ & $2 b$ & Kolovi \\
\hline 241.0718_7.45_147.9 & 241.0718 & 7.45 & 147.9 & $\begin{array}{c}{\left[\mathrm{M}-\mathrm{H}^{]-}\right.} \\
{[\mathrm{M}+\mathrm{H}]^{+}}\end{array}$ & 95.0509 & $\mathrm{C}_{11} \mathrm{H}_{14} \mathrm{O}_{6}$ & Elenolic acid ${ }^{4}$ & $2 b$ & Koroneiki \\
\hline 247.2066_10.11_180.2 & 247.2066 & 10.11 & 180.2 & {$[\mathrm{M}-\mathrm{H}]-$} & $\begin{array}{l}205.1591 \\
133.0650 \\
123.0790\end{array}$ & $\mathrm{C}_{17} \mathrm{H}_{28} \mathrm{O}$ & - & 4 & Kolovi \\
\hline 269.2484_14.03_269.5 & 269.2484 & 14.03 & 269.5 & $\begin{array}{c}{[\mathrm{M}-\mathrm{H}]^{-}} \\
{[\mathrm{M}+\mathrm{H}]^{+}}\end{array}$ & 262.8075 & $\mathrm{C}_{17} \mathrm{H}_{34} \mathrm{O}_{2}$ & $\begin{array}{l}\text { 11-Methylhexa } \\
\text { decanoic acid }^{1}\end{array}$ & 3 & Adramytiani \\
\hline 297.2434_11.94_305.6 & 297.2434 & 11.94 & 305.6 & {$[\mathrm{M}-\mathrm{H}]^{-}$} & N.F. & $\mathrm{C}_{18} \mathrm{H}_{34} \mathrm{O}_{3}$ & $\begin{array}{l}\text { 18-hydroxyoleate, }{ }^{1} \\
9,10 \text {-epoxystearate }\end{array}$ & 3 & Koroneiki \\
\hline 471.3478_12.58_222.9 & 471.3478 & 12.58 & 222.9 & {$[\mathrm{M}-\mathrm{H}]^{-}$} & N.F. & $\mathrm{C}_{30} \mathrm{H}_{48} \mathrm{O}_{4}$ & $\begin{array}{l}\text { Maslinic acid, }{ }^{1} \\
\text { Corosolic acid }\end{array}$ & 3 & Koroneiki \\
\hline 487.3424_11.16_226.7 & 487.3424 & 11.16 & 226.7 & {$[\mathrm{M}-\mathrm{H}]^{-}$} & N.F. & $\mathrm{C}_{30} \mathrm{H}_{48} \mathrm{O}_{5}$ & $\begin{array}{l}\text { Rotundic acid, }{ }^{1} \\
\text { Tormentic acid }\end{array}$ & 3 & Kolovi \\
\hline 271.0616_6.92_163.2 & 271.0616 & 6.92 & 163.2 & {$[\mathrm{M}-\mathrm{H}]^{-}$} & $\begin{array}{l}119.0500 \\
151.0035\end{array}$ & $\mathrm{C}_{15} \mathrm{H}_{12} \mathrm{O}_{5}$ & Naringenin $^{3}$ & 1 & Kolovi \\
\hline 205.1563_9.84_147.6 & 205.1563 & 9.84 & 147.6 & {$[\mathrm{M}+\mathrm{H}]^{+}$} & 107.0834 & $\mathrm{C}_{14} \mathrm{H}_{20} \mathrm{O}$ & $\begin{array}{l}\text { 3-Benzyl-4- } \\
\text { heptanone }\end{array}$ & $2 b$ & Koroneiki \\
\hline 357.1330_9.35_181.4 & 357.1330 & 9.35 & 181.4 & {$[\mathrm{M}+\mathrm{H}]^{+}$} & $\begin{array}{l}327.1197 \\
137.0577\end{array}$ & $\mathrm{C}_{27} \mathrm{H}_{16} \mathrm{O}$ & - & 4 & Koroneiki \\
\hline
\end{tabular}

${ }^{1}$ Annotated according to EVOOs suspect database. ${ }^{9}$

${ }^{2}$ Annotated according to workflow developed in "Integrated workflow for the identification of unknowns" section.

${ }^{3}$ Annotated according to EVOOs target database.

${ }^{4}$ Annotated according to OliveNet ${ }^{\mathrm{TM}}$ database. ${ }^{10}$

* N.F.: compound of no fragmentation 


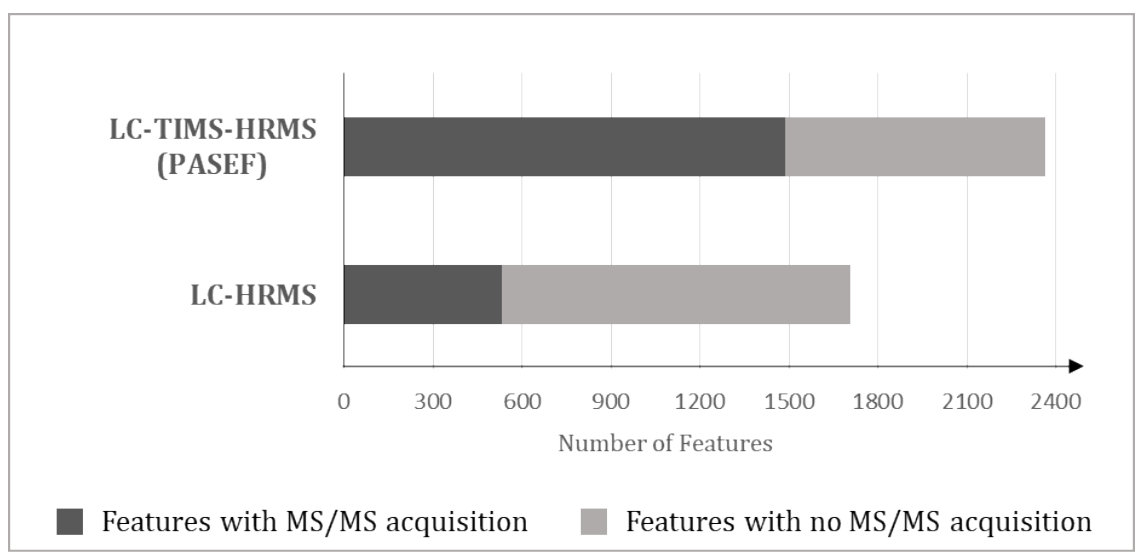

Figure S-12. Number of features detected in LC-HRMS empowered by PASEF and in LC-HRMS. TIMS-PASEF incorporation in the methodology results in an increase in features number, while MS/MS spectra acquisition is also amplified.

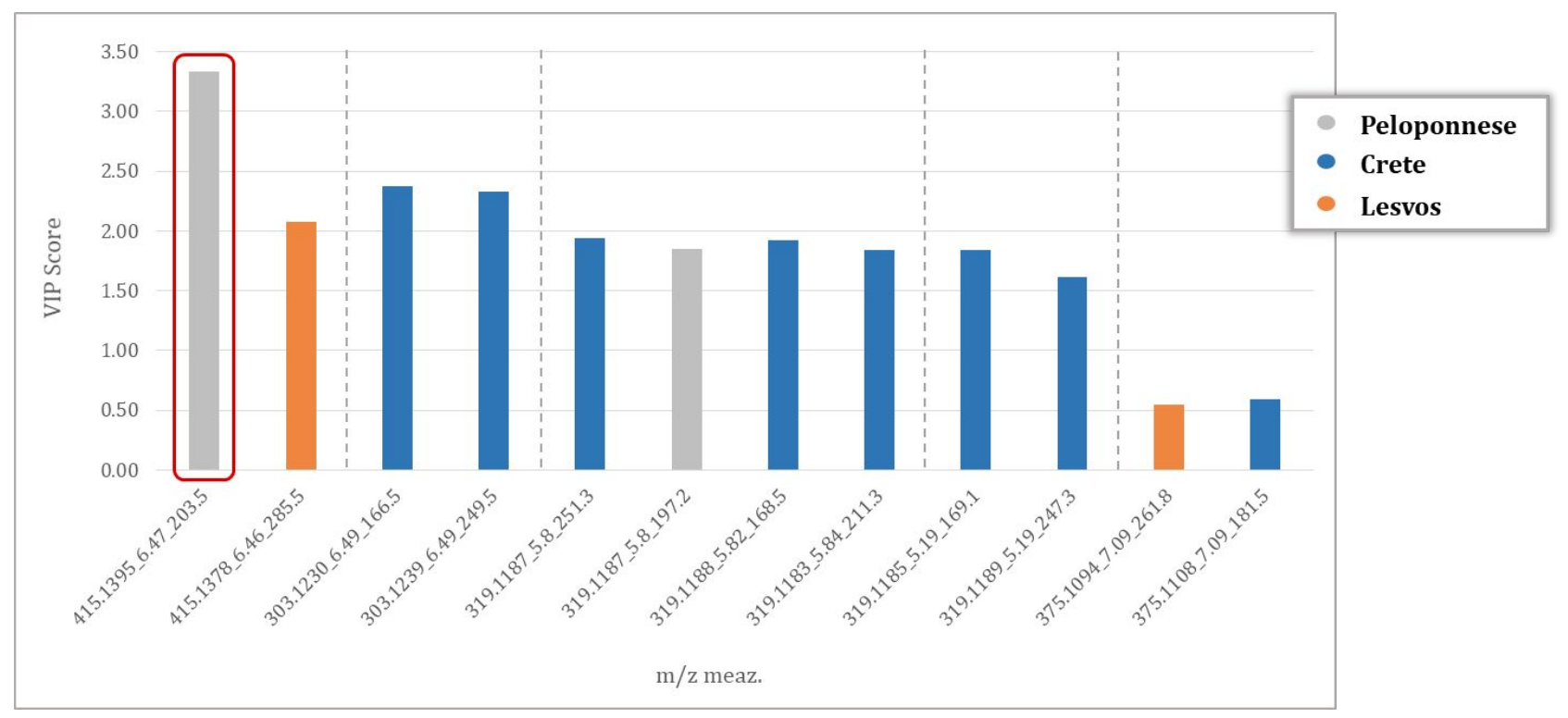

Figure S-13. Secoiridoid isomers - markers of geographical origin, detected in negative ionization mode in correspondence with their VIP score. In y-axis, features detected are coded according to the format "accurate mass $(\mathrm{m} / \mathrm{z})$ _retention time_CCS value", that corresponds to experimental measurements of each feature in particular. Circled in red is the feature with the major contribution in the achieved discrimination. 
Table S-6. Identified secoiridoid isomeric markers in EVOOs geographical origin discrimination study. Features detected are coded according to the format "accurate mass (m/z)_retention time_CCS value", that corresponds to experimental measurements of each feature in particular.

\begin{tabular}{|c|c|c|c|c|c|c|c|c|c|}
\hline Features & m/z meas. & $\begin{array}{c}\mathbf{t}_{\mathrm{R}} \\
(\mathrm{min})\end{array}$ & $\begin{array}{l}\text { CCS } \\
\left(\AA^{2}\right)\end{array}$ & Ions & Fragments & $\begin{array}{l}\text { Putative } \\
\text { Formula }\end{array}$ & Identification & $\begin{array}{c}\text { ID } \\
\text { Level }\end{array}$ & Marker \\
\hline 415.1395_6.47_203.5 & 415.1395 & 6.47 & 203.5 & {$[\mathrm{M}-\mathrm{H}]^{-}$} & $\begin{array}{l}151.0398 \\
181.0506 \\
136.0163 \\
325.1095\end{array}$ & $\mathrm{C}_{22} \mathrm{H}_{24} \mathrm{O}_{8}$ & 1-Acetoxypinoresinol ${ }^{1}$ & $2 b$ & Peloponnese \\
\hline 415.1378_6.46_285.5 & 415.1378 & 6.46 & 285.5 & {$[\mathrm{M}-\mathrm{H}]^{-}$} & $\begin{array}{l}151.0398 \\
181.0506 \\
136.0163 \\
325.1095\end{array}$ & $\mathrm{C}_{22} \mathrm{H}_{24} \mathrm{O}_{8}$ & 8-Acetoxypinoresinol ${ }^{1}$ & $2 b$ & Lesvos \\
\hline 303.1230_6.49_166.5 & 303.1230 & 6.49 & 166.5 & {$[\mathrm{M}-\mathrm{H}]^{-}$} & 137.0602 & $\mathrm{C}_{17} \mathrm{H}_{20} \mathrm{O}_{5}$ & Oleocanthal isomer $^{2}$ & $2 b$ & Crete \\
\hline 303.1239_6.49_249.5 & 303.1239 & 6.49 & 249.5 & {$[\mathrm{M}-\mathrm{H}]^{-}$} & 137.0602 & $\mathrm{C}_{17} \mathrm{H}_{20} \mathrm{O}_{5}$ & Oleocanthal $^{2}$ & 1 & Crete \\
\hline 319.1187_5.8_251.3 & 319.1187 & 5.80 & 251.3 & {$[\mathrm{M}-\mathrm{H}]^{-}$} & $\begin{array}{l}183.0656 \\
195.0656\end{array}$ & $\mathrm{C}_{17} \mathrm{H}_{20} \mathrm{O}_{6}$ & Oleacein $^{2}$ & 1 & Crete \\
\hline 319.1187_5.8_197.2 & 319.1187 & 5.80 & 197.2 & {$[\mathrm{M}-\mathrm{H}]^{-}$} & $\begin{array}{l}183.0656 \\
195.0656\end{array}$ & $\mathrm{C}_{17} \mathrm{H}_{20} \mathrm{O}_{6}$ & Oleacein isomer $^{2}$ & $2 b$ & Peloponnese \\
\hline 319.1188_5.82_168.5 & 319.1188 & 5.82 & 168.5 & {$[\mathrm{M}-\mathrm{H}]^{-}$} & $\begin{array}{l}183.0656 \\
195.0656\end{array}$ & $\mathrm{C}_{17} \mathrm{H}_{20} \mathrm{O}_{6}$ & Oleacein isomer $^{2}$ & $2 b$ & Crete \\
\hline 319.1183_5.84_211.3 & 319.1183 & 5.84 & 211.3 & {$[\mathrm{M}-\mathrm{H}]^{-}$} & $\begin{array}{l}183.0656 \\
195.0656\end{array}$ & $\mathrm{C}_{17} \mathrm{H}_{20} \mathrm{O}_{6}$ & Oleacein isomer $^{2}$ & $2 b$ & Crete \\
\hline 319.1185_5.19_169.1 & 319.1185 & 5.19 & 169.1 & {$[\mathrm{M}-\mathrm{H}]^{-}$} & $\begin{array}{l}199.0608 \\
111.0081\end{array}$ & $\mathrm{C}_{17} \mathrm{H}_{20} \mathrm{O}_{6}$ & Oleocanthalic acid ${ }^{2}$ & $2 b$ & Crete \\
\hline 319.1189_5.19_247.3 & 319.1189 & 5.19 & 247.3 & {$[\mathrm{M}-\mathrm{H}]^{-}$} & $\begin{array}{l}199.0608 \\
111.0081\end{array}$ & $\mathrm{C}_{17} \mathrm{H}_{20} \mathrm{O}_{6}$ & Oleocanthalic acid ${ }^{2}$ & 1 & Crete \\
\hline 375.1094_7.09_261.8 & 375.1094 & 7.09 & 261.8 & {$[\mathrm{M}-\mathrm{H}]^{-}$} & $\begin{array}{l}195.0657 \\
153.0556 \\
127.0398\end{array}$ & $\mathrm{C}_{20} \mathrm{H}_{24} \mathrm{O}_{7}$ & $\begin{array}{c}\text { Dehydro - Oleuropein } \\
\text { aglycone }^{3}\end{array}$ & 3 & Lesvos \\
\hline 375.1108_7.09_181.5 & 375.1108 & 7.09 & 181.5 & {$[\mathrm{M}-\mathrm{H}]^{-}$} & $\begin{array}{l}195.0657 \\
153.0556 \\
127.0398\end{array}$ & $\mathrm{C}_{20} \mathrm{H}_{24} \mathrm{O}_{7}$ & $\begin{array}{c}\text { Dehydro - Oleuropein } \\
\text { aglycone }^{3}\end{array}$ & 3 & Crete \\
\hline
\end{tabular}

${ }^{1}$ Annotated according to workflow developed in "Integrated workflow for the identification of unknowns" section.

${ }^{2}$ Annotated according to EVOOs target database.

${ }^{3}$ Annotated according to EVOOs suspect database. ${ }^{9}$ 
(A)

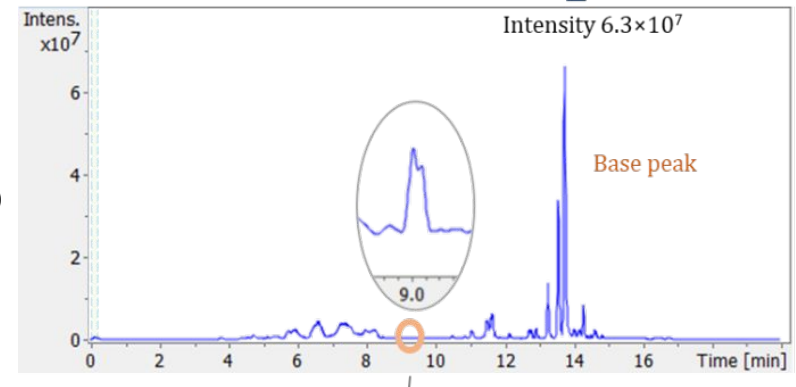

(B)

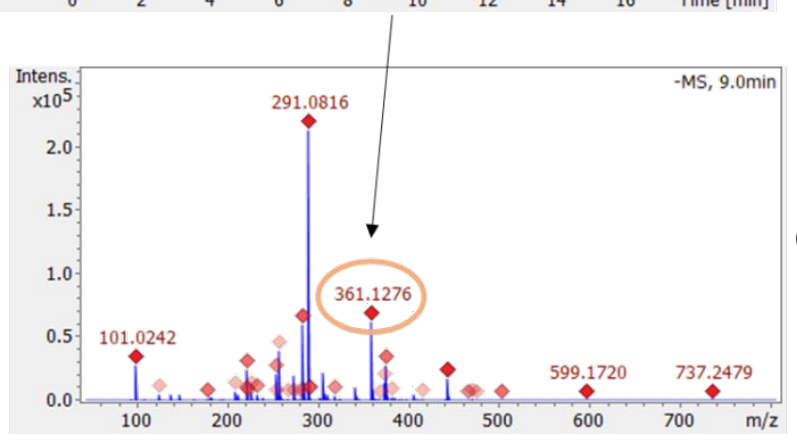

$1000 \times$ lower

(C)

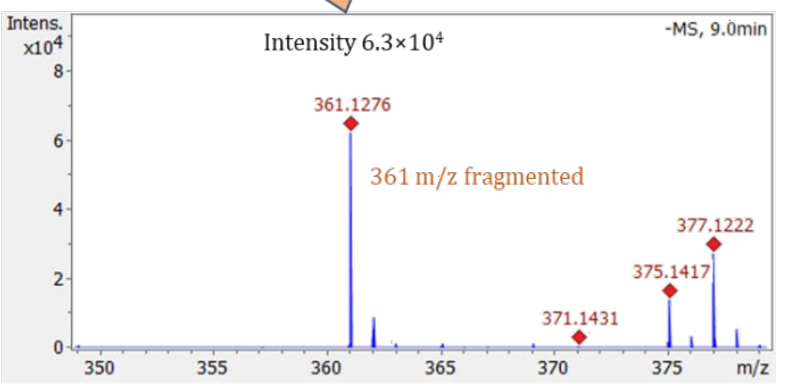

(D)

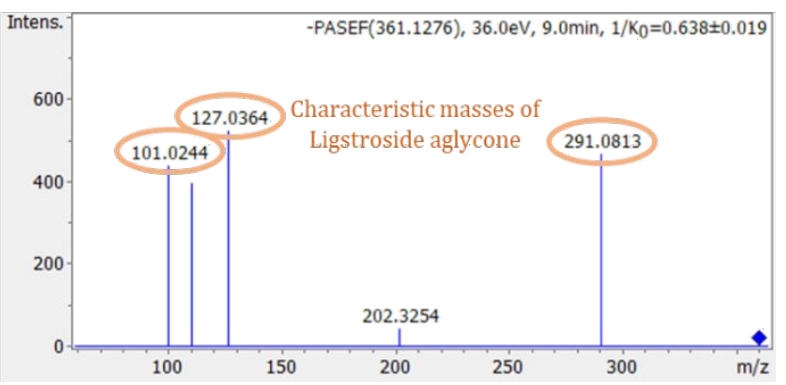

Figure S-14. Characteristic example of Ligstroside aglycone (accurate $\mathrm{m} / \mathrm{z}$ 361.1293) indicating the high sensitivity achieved through PASEF acquisition mode. Selecting a particular retention time range in Base Peak Chromatogram (BPC) in which the compound elutes (A), a large number of precursor ions are detected, Ligstroside aglycone included (B). The precursor ion of Ligstroside aglycone is selected by the quadrupole mass filter, even at 3 order of magnitude lower that the most abundant ion of the BPC (C). Reliable MS/MS spectrum of the compound is acquired, with compound's main fragments being detected even at low intensities (D). 


\section{SI-9 Integrated workflow for the identification of unknowns}

For rapid feature assessment, intensity of the feature in each sample of the data set is being visualized through MetaboScape software, both chromatographically and in the mobilogram part (Figure S-15). More specifically box plot for this particular feature is exported in order to quick evaluate its contribution to discrimination.

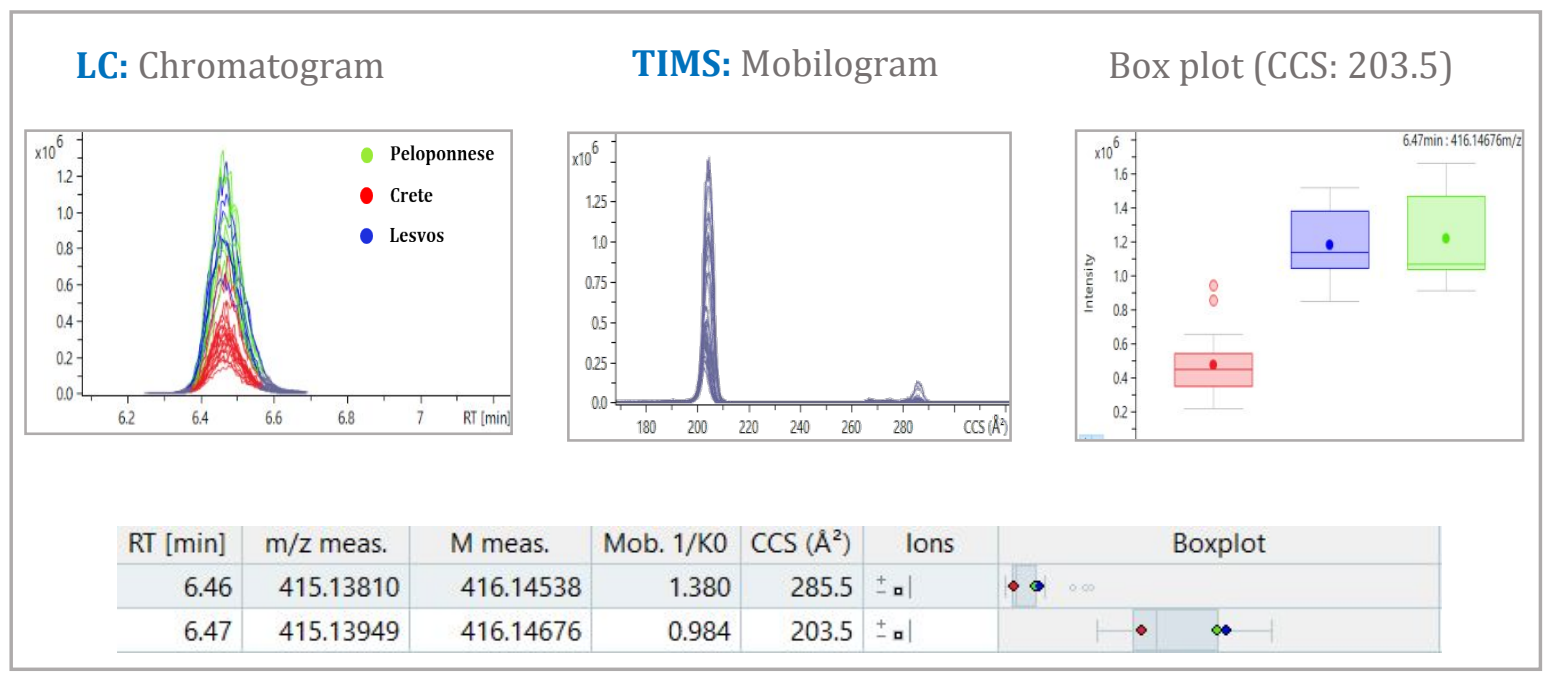

Figure S-15. Primary information for the two features, differentiated by their mobilities, as retrieved from MetaboScape bucket table. Chromatographic and mobility information of the feature is provided, while quick visualization of feature contribution in the discrimination is achieved through box plot chart.

As a first step to assess their molecular formula(s), SmartFormula (SF) 3D tool was applied (Figure S-16). To limitate candidates, a threshold of $5 \mathrm{mDa}$ tolerance was set during search. Isotope pattern visualization of this particular formula is also enabled and depicted.

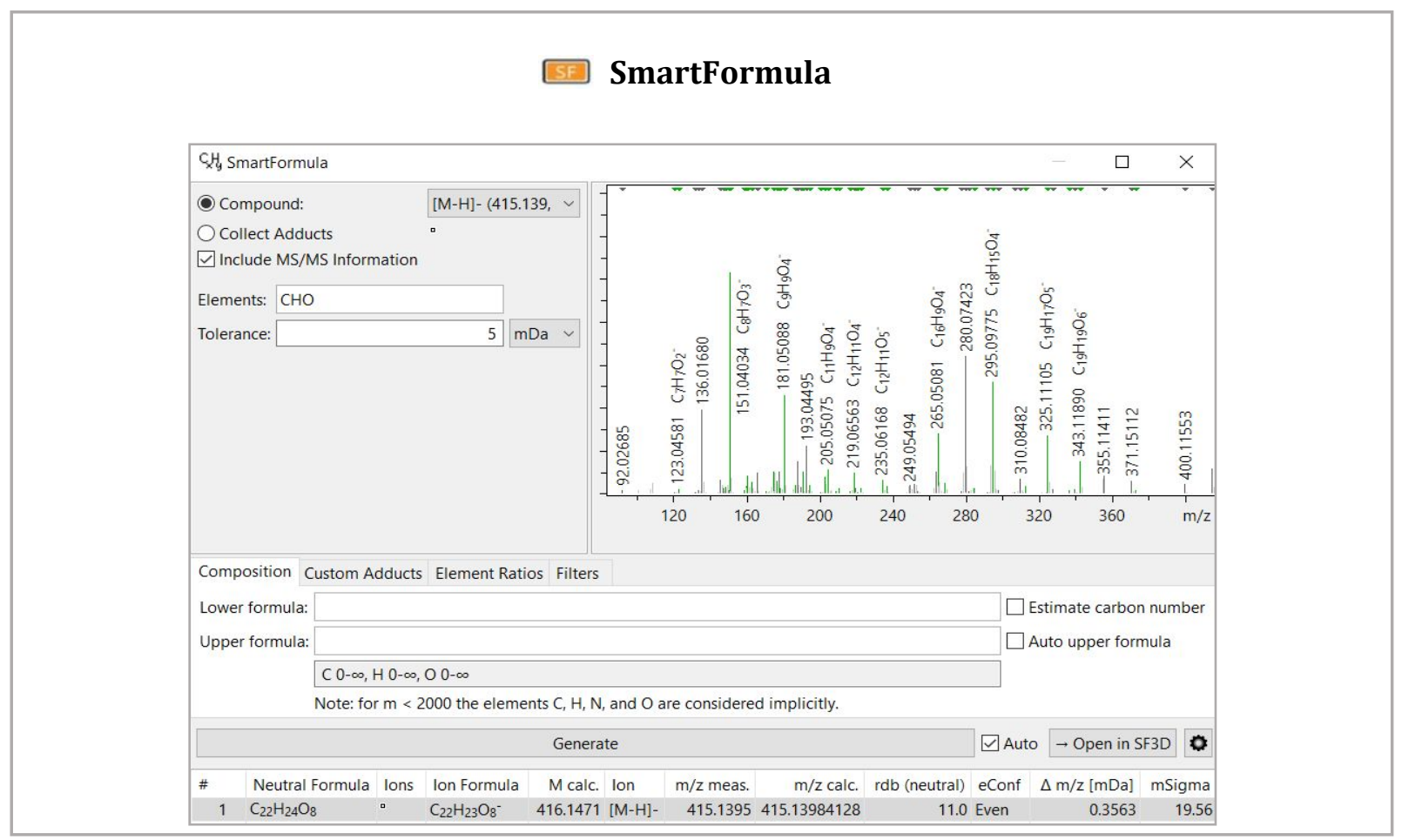

Figure S-16. SmartFormula implementation for molecular formula assignment. SF3D takes into account all information of MS and MS/MS data, thus reducing significantly the number of possible candidates that comply with the matching criteria. Focusing on the class of bioactive compounds, the search is limited to compounds consisted exclusively of carbon, hydrogen and oxygen. 
Compound Crawler was used for structure assessment. Compounds structures were searched via PubChem database. Two spatial isomers, 1- and 8-Acetoxypinoresinol (with their structural difference highlighted in orange), prevailed in the search as bioactive compounds, similar in structure with compounds already detected and identified in EVOOs (Figure S-17).

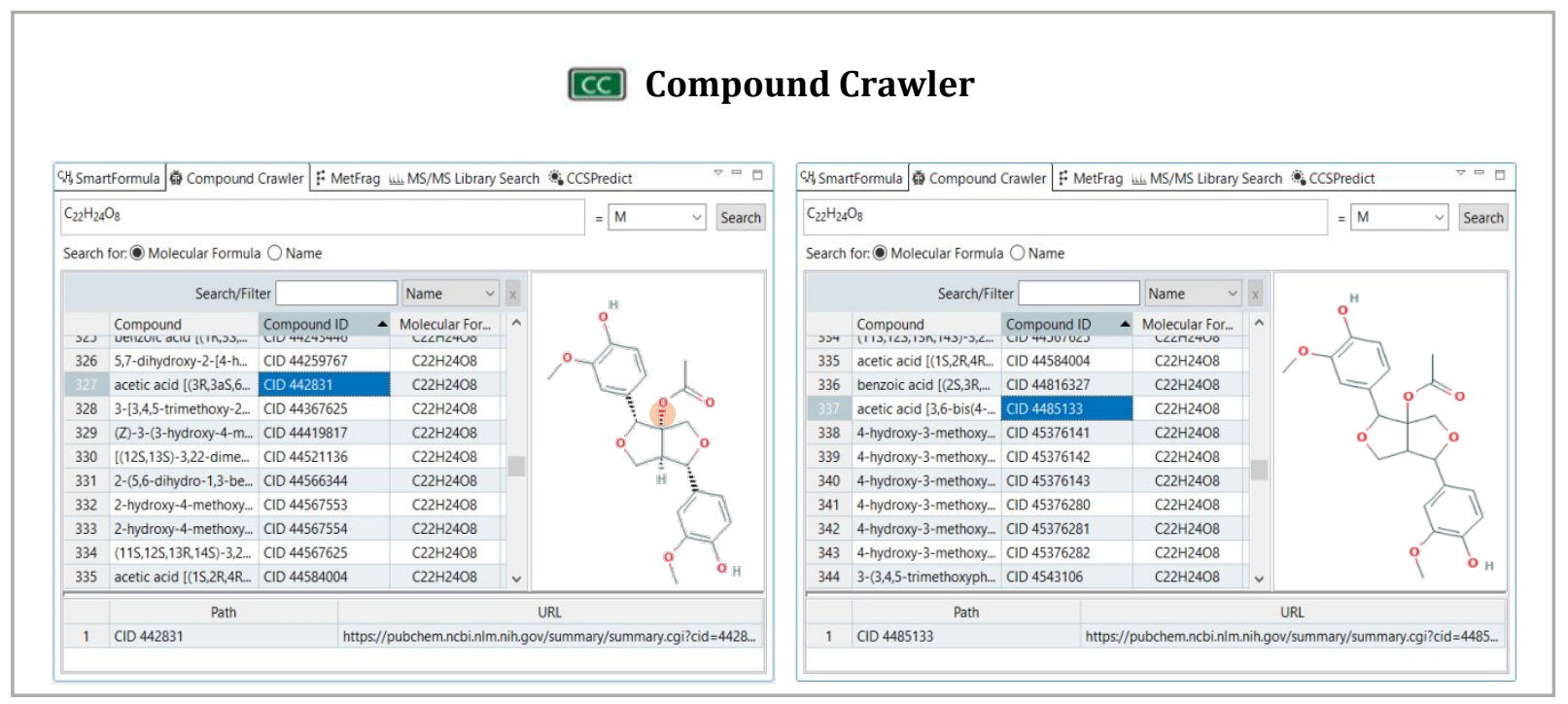

Figure S-17. Structure annotation was achieved via online or personal databases search, using Compound Crawler tool. 2 potential isomers are prevailed in the online database search, highlighted as spatial isomers.

The two compounds best explained all in-silico fragments, as these were exported through via MetFrag (MF) (Figure S-18). Both compounds fragmentation pattern was identical in MF due to their high structural similarity. A relative score is also provided through MF tool that signifies fragmentation matching. In our example, all fragments were successfully interpreted, thus the ultimate score of 1.0/1.0 was exported. The explained fragment peaks are visualized in the MS/MS spectrum, as well as the molecular structure with sub-structure highlighting. 


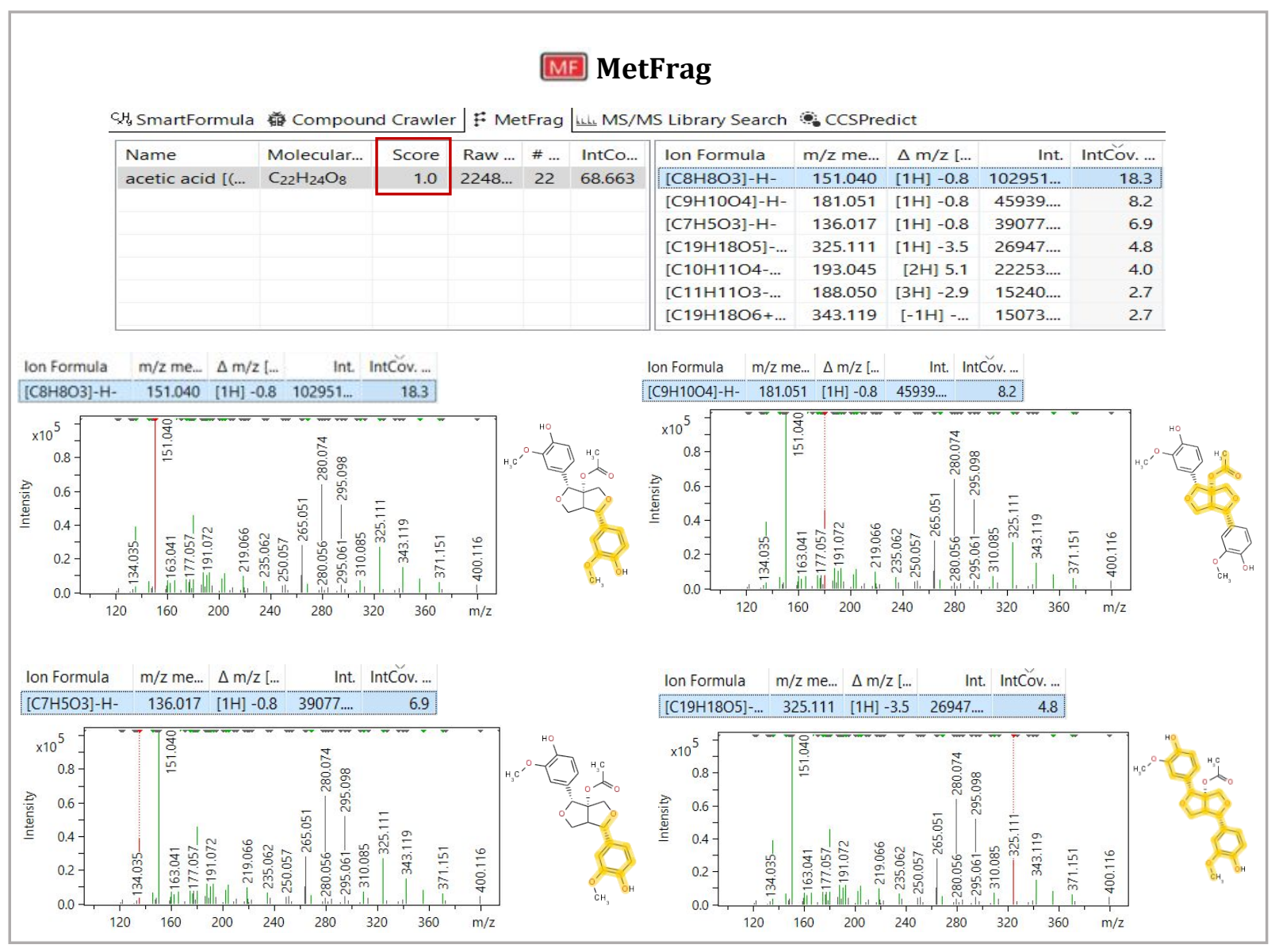

Figure S-18. In silico fragmentation of the compound performed using MetFrag application tool. Each fragment is pinpointed in the MS2 spectrum with a vertical red line, while the fragment is also highlighted in yellow upon compound's structure. The proposed compound best explains all fragments, recording the best matching score 1.0 (highlighted in red). 


\section{REFERENCES}

(1) Diamantakos, P.; Velkou, A.; Killday, K. B.; Gimisis, T.; Melliou, E.; Magiatis, P. Oleokoronal and Oleomissional: New Major Phenolic Ingredients of Extra Virgin Olive Oil. Olivae 2015, 122 (August 2016), 22-32.

(2) Ogata, K.; Ishihama, Y. Extending the Separation Space with Trapped Ion Mobility Spectrometry Improves the Accuracy of Isobaric Tag-Based Quantitation in Proteomic LC/MS/MS. Anal. Chem. 2020, 92 (12), 8037-8040. https://doi.org/10.1021/acs.analchem.0c01695.

(3) Picache, J. A.; Rose, B. S.; Balinski, A.; Leaptrot, K. L.; Sherrod, S. D.; May, J. C.; McLean, J. A. Collision Cross Section Compendium to Annotate and Predict Multi-Omic Compound Identities. Chem. Sci. 2019, 10 (4), 983-993. https://doi.org/10.1039/c8sc04396e.

(4) Vautz, W.; Zimmermann, D.; Hartmann, M.; Baumbach, J. I.; Nolte, J.; Jung, J. Ion Mobility Spectrometry for Food Quality and Safety. Food Addit. Contam. 2006, 23 (11), 1064-1073. https://doi.org/10.1080/02652030600889590.

(5) Schroeder, M.; Meyer, S. W.; Heyman, H. M.; Barsch, A.; Sumner, L. W. Generation of a Collision Cross Section Library for Multi-Dimensional Plant Metabolomics Using UHPLC-Trapped Ion Mobility-MS/MS. Metabolites 2020, 10 (1). https://doi.org/10.3390/metabo10010013.

(6) Causon, T. J.; Ivanova-Petropulos, V.; Petrusheva, D.; Bogeva, E.; Hann, S. Fingerprinting of Traditionally Produced Red Wines Using Liquid Chromatography Combined with Drift Tube Ion Mobility-Mass Spectrometry. Anal. Chim. Acta 2019 1052, 179-189. https://doi.org/10.1016/j.aca.2018.11.040.

(7) Abbattista, R.; Losito, I.; De Ceglie, C.; Basile, G.; Calvano, C. D.; Palmisano, F.; Cataldi, T. R. I. Structural Characterization of the Ligstroside Aglycone Isoforms in Virgin Olive Oils by Liquid Chromatography-High-Resolution Fourier-Transform Mass Spectrometry and H/Dexchange. J. Mass Spectrom. 2019, 54 (10), 843-855. https://doi.org/10.1002/jms.4438.

(8) Celma, A.; Sancho, J. V.; Schymanski, E. L.; Fabregat-Safont, D.; Ibáñez, M.; Goshawk, J.; Barknowitz, G.; Hernández, F.; Bijlsma, L. Improving Target and Suspect Screening High-Resolution Mass Spectrometry Workflows in Environmental Analysis by Ion Mobility Separation. Environ. Sci. Technol. 2020. https://doi.org/10.1021/acs.est.0c05713.

(9) Kalogiouri, N. P.; Aalizadeh, R.; Thomaidis, N. S. Application of an Advanced and Wide Scope Non-Target Screening Workflow with LC-ESI-QTOF-MS and Chemometrics for the Classification of the Greek Olive Oil Varieties. Food Chem. 2018, 256 (July 2017), 53-61. https://doi.org/10.1016/j.foodchem.2018.02.101.

(10) Bonvino, N. P.; Liang, J.; McCord, E. D.; Zafiris, E.; Benetti, N.; Ray, N. B.; Hung, A.; Boskou, D.; Karagiannis, T. C. OliveNet $^{\mathrm{TM}}$ : A Comprehensive Library of Compounds from Olea Europaea. Database 2018, 2018 (2018), 1-9. https://doi.org/10.1093/database/bay016. 